\title{
Reanalyzing an evaporating black hole
}

\author{
Yasunori Nomura \\ Berkeley Center for Theoretical Physics, Department of Physics, \\ University of California, Berkeley, California 94720, USA, \\ Theoretical Physics Group, Lawrence Berkeley National Laboratory, Berkeley, California 94720, USA, \\ and Kavli Institute for the Physics and Mathematics of the Universe (WPI), UTIAS, \\ The University of Tokyo, Kashiwa, Chiba 277-8583, Japan
}

(Received 29 October 2018; published 8 April 2019)

\begin{abstract}
A coherent picture of the quantum mechanics of a collapse-formed, evaporating black hole is presented. In a distant frame, semiclassical theory in the zone describes microscopic dynamics of only the "hard modes," the modes that are hard enough to be discriminated in the timescale of Hawking emission. The thermal nature of these modes arises from microcanonical typicality of the full black hole degrees of freedom, mostly composed of the "soft modes," the modes that cannot be discriminated at the semiclassical level. The hard modes are purified by a combined system of the soft modes and early Hawking radiation but not by either of them separately. This intrinsically tripartite structure of entanglement is general, regardless of the age of the black hole. The interior spacetime emerges only at a coarse-grained level. To describe it, an effective theory can be erected at each time, which applies only to a limited spacetime region determined by the time at which the theory is erected. The entire interior of the black hole can be described only using multiple effective theories erected at different times, realizing the idea of complementarity. We analyze the implications of the entanglement structure described here for various phenomena, including Hawking evaporation and general information retrieval. For multiple entangled black holes, it implies that semiclassical objects dropped into different black holes cannot meet in the interior, although each object smoothly enters the horizon of the black hole to which it is falling. We also discuss physics in Rindler space, elucidating how it is obtained as a smooth limit of the black hole physics.
\end{abstract}

DOI: $10.1103 /$ PhysRevD.99.086004

\section{INTRODUCTION}

A black hole is an object in general relativity from which nothing can escape. As in any other object, its entropy, formally defined as the logarithm of the number of independent states in a fixed energy interval, is infinity at the classical level $S_{\mathrm{cl}}=\infty$, which would give zero temperature $T_{\mathrm{cl}}=\left(\partial S_{\mathrm{cl}} / \partial E\right)^{-1}=0$. Quantum mechanics, however, regulate these to give [1-3]

$$
S(M)=\frac{\mathcal{A}(M)}{4 l_{\mathrm{P}}^{2}}, \quad T(M)=\frac{1}{8 \pi M l_{\mathrm{P}}^{2}},
$$

where $M$ and $\mathcal{A}(M)=16 \pi M^{2} l_{\mathrm{p}}^{4}$ are the mass and horizon area of a (Schwarzschild) black hole, respectively, $l_{\mathrm{P}}$ is the Planck length, and we have adopted natural units $c=\hbar=1$. A surprising thing is that the entropy is proportional to the surface area rather than the volume,

Published by the American Physical Society under the terms of the Creative Commons Attribution 4.0 International license. Further distribution of this work must maintain attribution to the author(s) and the published article's title, journal citation, and DOI. Funded by SCOAP . which has led to the idea that a fundamental theory of quantum gravity is formulated holographically in nondynamical, lower-dimensional spacetime [4-6].

The fact that a black hole radiates, and so eventually evaporates, allows us to understand it as a resonance appearing, e.g., in an intermediate stage of a scattering process $[7,8]$. (For an extremal black hole, this requires a conjectured property of quantum gravity [9].) In fact, the anti-de Sitter (AdS)/conformal field theory (CFT) correspondence [10-12] strongly suggests that formation and evaporation of a black hole occurs unitarily, making the concern of information loss [13] obsolete. The unitary evolution of a black hole, however, raises another issue of quantum information cloning [14,15]: If Hawking radiation contains full information about an object that has fallen into a black hole earlier, then its simultaneous existence with the object in the interior spacetime would imply a cloning of quantum information, which is forbidden by linearity of quantum mechanics [16]. An interesting idea addressing this issue is called complementarity [17], which asserts that information about the interior spacetime is not independent of that in Hawking radiation. The explicit realization of this idea, however, has not been clear, and there are even 
arguments that it cannot be implemented consistently with the usual postulates of semiclassical physics [18-20].

The purpose of this paper is to reanalyze quantum mechanics of a collapse-formed, evaporating black hole, given recent developments. We begin with a series of assumptions that seem reasonable and are consistent with our latest understanding of the subject and then develop a coherent picture from them. Essences of the resulting picture involve those discussed in Refs. [21-23] and Refs. [24-26]. Semiclassical theory in a black hole background describes only a small subset of the fundamental degrees of freedom (d.o.f.), which are distributed nonlocally throughout the zone region [21-23]. The interior spacetime appears after coarse graining microscopic d.o.f. in a state-dependent manner [24-26]. The picture also contains an element of Ref. [27] in that the relevant microscopic d.o.f. involve those of early Hawking radiation, although the structure of entanglement we find differs from that considered there.

While some of the concepts used have been developed in the context of AdS/CFT, we will avoid the language of holography as much as possible, since the question is mostly about the microscopic picture in the bulk. Our focus will be on Schwarzschild black holes in four-dimensional asymptotically flat spacetime (or small black holes in four-dimensional asymptotically AdS spacetime). However, the restriction on specific spacetime dimensions or on nonrotating, noncharged black holes is not essential for our discussion.

In the description based on a distant reference frame, our picture has the following key features:

(i) In the black hole zone region, semiclassical theory describes microscopic dynamics of only the hard modes, the modes that are hard enough to be discriminated in the timescale of Hawking emission. These modes comprise only a tiny fraction of the total black hole d.o.f. The other, soft modes can be described only statistically.

(ii) The thermal nature of the hard modes arises because they are in equilibrium with the soft modes, the vast majority of the black hole d.o.f. In particular, the canonical nature of the hard (semiclassical) modes arises from the microcanonical ensemble of the full black hole d.o.f.

(iii) The spatial distribution of the soft modes can be defined by interactions with the other modes, and it is given by the entropy density determined by the local Hawking temperature $T(M) / \sqrt{1-2 M l_{\mathrm{P}}^{2} / r}$. While this distribution is strongly peaked toward the stretched horizon, there are $O(1)$ numbers of d.o.f. located around the edge of the zone. Although these comprise only a tiny portion-fractionally of $O\left(l_{\mathrm{P}}^{2} / \mathcal{A}(M)\right)$ - of the full black hole d.o.f., they play an important role in Hawking emission.

(iv) The hard modes in the zone region are purified by a combined system of the soft modes and early Hawking radiation but not by either of them separately. In particular, the correlation between the hard modes and either of the soft modes or early Hawking radiation is essentially classical. This is the case regardless of the age of the black hole, i.e., if it is younger or older than the Page time [8].

In the distant frame description, the evolution of a black hole is unitary, and the interior of the black hole is absent. In the context of holography, this corresponds to the description based on boundary time evolution [28].

The interior spacetime emerges only effectively at a coarse-grained level. The resulting effective theories have the following features:

(i) An effective theory can be erected at each time (of a distant description) for the purpose of describing a small object falling inside the horizon until it hits the singularity.

(ii) Each effective theory describes only a limited spacetime region determined by the time $t_{*}$ at which the effective theory is erected. Specifically, the region is the domain of dependence of the union of the zone and its mirror regions of a two-sided black hole obtained from the original black hole at $t_{*}$.

(iii) The mirror operators needed for an effective theory act on both the soft mode and early radiation d.o.f. In particular, neither of the soft modes nor early radiation alone can play the role of the second exterior of the effective two-sided description.

(iv) Since the spacetime region described by each effective theory is limited, the entire interior of a black hole can be covered only using multiple effective theories erected at different times, which are generally not mutually independent. This provides a specific way in which the idea of complementarity is implemented. It also provides a simple solution to the cloning paradox that no duplicate information occurs in any single description.

The entanglement structure between the hard modes, soft modes, and early radiation described above is intrinsically tripartite and, in a sense, is reminiscent of the GreenbergerHorne-Zeilinger (GHZ) form [29]. It implies, together with a simple assumption about the dynamics of the black hole, that manipulating early Hawking radiation alone cannot destroy a smooth horizon of the black hole. It also implies that a pair of entangled Schwarzschild (or small AdS) black holes are not connected causally by a wormhole; namely, objects dropped into different black holes cannot meet in the interior spacetime. The situation, therefore, is different from that in Refs. [30,31], which consider entangled large AdS black holes in a thermal state.

In addition to analyzing physics of an evaporating black hole, we also consider the Rindler limit. This elucidates the relation between Hawking emission/mining [32,33] from a black hole and Unruh radiation [34-36] seen by an accelerating observer in Minkowski space. In particular, it clarifies "information flow" associated with the Unruh effect. 


\section{A. Relation to other work}

The physics of an evaporating black hole has been studied in a large amount of literature, especially after the work of Refs. [18-20], some of which overlap with the picture presented here at conceptual levels. The fact that the d.o.f. described by semiclassical theory comprise only a tiny fraction of the entire d.o.f. was emphasized in Ref. [37], which was later demonstrated in a more convincing form in Ref. [38]. Nonlocality associated with the Hawking emission process was considered in Refs. [39-41], although here we do not need a deviation from local dynamics at the semiclassical level. Nonlocality of Hawking emission more similar to the one discussed here [21-23] was noted in Refs. [42-45]. State dependence of interior operators [24-26] was also considered in Refs. [46-50]. Earlier attempts to avoid firewalls along the lines of Ref. [27] include Refs. [51-54]. For more recent analyses, see Refs. [55-57].

\section{B. Outline}

In Sec. II, we study an evaporating black hole as viewed from a distant observer. In Sec. II A, we discuss black hole microstates and introduce the concept of the hard and soft modes. In Sec. II B, we describe how the thermal nature of a black hole emerges from a microscopic point of view, which elucidates what semiclassical theory is. In Sec. II C, we analyze the Hawking emission process. We discuss how information is transferred from a black hole to ambient space, emphasizing that the nonlocal distribution of black hole information plays an important role. We find that entanglement between the hard mode, soft mode, and early radiation d.o.f. takes an intrinsically tripartite form, regardless of the age of the black hole.

In Sec. III, we discuss how the picture of the interior spacetime emerges from the microscopic point of view. In Sec. III A, we study basic kinematics, emphasizing that the equivalence principle dictates the dynamics of only a small object, which is well described by the hard modes. In Sec. III B, we discuss how the effective two-sided description may emerge through coarse graining from the entanglement structure discussed in Sec. II. This allows us to erect an effective theory of the interior at each time of a distant description. While each theory erected in this way covers only a limited portion of the interior spacetime, the full picture of the interior can be obtained (only) with a collection of effective theories. We also argue that our framework provides the "simplest" solution to the cloning paradox: No duplication of information occurs in any single description, regardless of whether it can be operationally possessed by an observer or not.

In Sec. IV, we discuss the Rindler limit, aiming to clarify the relation between Hawking emission/mining from a black hole and the Unruh effect in Minkowski space. In Sec. V, we consider multiple black holes and see that the situation of entangled Schwarzschild (or small AdS) black holes is different from that of entangled large AdS black holes in a thermal state. Finally, we conclude in Sec. VI, in which we discuss implications for a holographic description and make a few general remarks about the black hole interior and singularity.

\section{BLACK HOLE AND INFORMATION}

In this section, we discuss how a collapse-formed, or single-sided, black hole can be described from the viewpoint of a distant observer. We discuss the interpretation of the Bekenstein-Hawking entropy and how the d.o.f. it represents interact with the modes described by semiclassical theory. We also discuss implications of this picture for the Hawking emission process, including the evolution of the entanglement structure during evaporation. Throughout, we assume that the evolution of a black hole is unitary in a distant description.

\section{A. Black hole microstates}

Consider a set of states having energies, as measured in the asymptotic region, between $E$ and $E+\Delta E$. Some of these states can be recognized from the asymptotic region as those representing multiple particle excitations of masses $m_{i}: \sum_{i} m_{i} \approx E$. Such a decomposition is possible if these particles are distributed with sufficiently large distances between them. There are, however, states in which this decomposition cannot be made completely. These include states having black holes. (They also include states having coherent excitations.) For simplicity, we will focus on states that have a single Schwarzschild black hole.

Let us consider a quantum state representing a black hole of mass $M$ located at some place at rest, where the position and velocity are measured with respect to a distant reference frame. Because of the uncertainty principle, such a state must involve a superposition of energy and momentum eigenstates. According to the standard Hawking calculation, a state of a black hole of mass $M$ will evolve after Schwarzschild time $t_{\mathrm{H}} \approx O\left(M l_{\mathrm{P}}^{2}\right)$ into a state representing a Hawking quantum of energy $\approx O\left(1 / M l_{\mathrm{P}}^{2}\right)$ and a black hole with the correspondingly smaller mass. ${ }^{1}$ The fact that these two states-before and after the emission-are nearly orthogonal implies that the original state must involve a superposition of energy eigenstates with a spread, at least, of

$$
\Delta E \approx \frac{1}{t_{\mathrm{H}}} \approx O\left(\frac{1}{M l_{\mathrm{P}}^{2}}\right) .
$$

Black hole states within this energy range cannot be discriminated in the asymptotic region because such a discrimination would require time longer than $t_{\mathrm{H}}$, the timescale with which a black hole state changes to another,

\footnotetext{
${ }^{1}$ Note that despite the apparent language here, the evolution of the state is continuous. Specifically, when the state is expanded in the eigenbasis of Hawking quanta, dominant terms of the state shift continuously to those with one more Hawking quantum with the characteristic timescale of $O\left(M l_{\mathrm{P}}^{2}\right)$.
} 
orthogonal state. These states, therefore, comprise the microstates of a black hole of mass $M$.

What about the spread of momentum $\Delta p$, with $p$ measured in the asymptotic region? Let us assume that the spatial location of the black hole is identified with precision comparable to the quantum stretching of the horizon $\Delta d \approx O\left(l_{\mathrm{s}}\right)$, namely, $\Delta r \approx O\left(l_{\mathrm{s}}^{2} / M l_{\mathrm{P}}^{2}\right)$, where $d$ and $r$ are the proper length and the Schwarzschild radial coordinate, respectively, and $l_{\mathrm{s}}$ is the string (cutoff) length. This implies that a black hole state must involve a superposition of momentum eigenstates with spread $\Delta p \approx\left(l_{\mathrm{s}} / M l_{\mathrm{P}}^{2}\right)(1 / \Delta d) \approx O\left(1 / M l_{\mathrm{P}}^{2}\right)$. Here, the factor $l_{\mathrm{s}} / M l_{\mathrm{P}}^{2}$ in the middle expression is the redshift factor. This value of $\Delta p$ corresponds to an uncertainty of the kinetic energy $\Delta E_{\mathrm{kin}} \approx(\Delta p)^{2} / M \approx O\left(1 / M^{3} l_{\mathrm{P}}^{4}\right)$, which is much smaller than $\Delta E$ in Eq. (2). The spread of energy, therefore, comes mostly from a superposition of different rest masses: $\Delta E \approx \Delta M$.

The number of microstates for a black hole is given by the Bekenstein-Hawking formula. Specifically, the number of independent microstates $\mathcal{N}(M)$ for a black hole of mass $M$ is given by

$$
\mathcal{N}(M)=\exp \left[\frac{\mathcal{A}(M)}{4 l_{\mathrm{P}}^{2}}\right] \frac{\Delta M}{M} \equiv e^{S_{\mathrm{BH}}(M)} \frac{\Delta M}{M},
$$

where

$$
\mathcal{A}(M)=16 \pi M^{2} l_{\mathrm{P}}^{4}
$$

is the area of the black hole horizon. The width of the mass range $\Delta M$ is given by Eq. (2), although the value of the Bekenstein-Hawking entropy $S_{\mathrm{BH}}(M)$ is insensitive to the precise choice of $\Delta M$, as in usual statistical mechanical systems.

The discussion above implies that it is not appropriate to consider that quantum mechanics introduces exponentially large degeneracies for black hole microstates which did not exist in a classical black hole. In classical general relativity, a set of Schwarzschild black holes located at some place at rest are parametrized by a continuous mass parameter $M$; i.e., there are a continuously infinite number of black hole states in the energy interval between $M$ and $M+\Delta M$ for any $M$ and small $\Delta M$. Quantum mechanics reduces this to a finite number $\approx e^{S_{\mathrm{BH}}(M)} \Delta M / M .^{2}$ This can also be seen from the fact that $S_{\mathrm{BH}}(M)$ is written as $\mathcal{A}(M) c^{3} / 4 \hbar l_{\mathrm{P}}^{2}$ when $c$ and $\hbar$ are restored, which becomes infinite for $\hbar \rightarrow 0$ with $c$ and $l_{\mathrm{P}}$ fixed. Indeed, this situation is quite standard in the

\footnotetext{
${ }^{2}$ Of course, quantum mechanics allows for a superposition of these finite number of independent states, so the number of possible (not necessarily independent) states is continuously infinite. The statement here applies to the number of independent states regarding classical black holes with different $M$ 's as independent states.
}

relation between quantum and classical mechanics. For example, the number of independent states of a harmonic oscillator in a fixed energy interval is finite in quantum mechanics (labeled by a discrete number for the levels), while it is infinite in classical mechanics (labeled by a continuous amplitude).

\section{B. Semiclassical description and the static background approximation}

From now on, we will suppress the location of a black hole and write a quantum state containing a black hole of mass $M$ as

$$
|\Psi(M)\rangle \approx|\psi(M)\rangle|\phi\rangle,
$$

where $|\psi(M)\rangle$ represents the state of the system within the zone region $r \leq r_{\mathrm{z}} \approx 3 M l_{\mathrm{P}}^{2}$, while $|\phi\rangle$ represents the state of the far region $r>r_{\mathrm{z}}$. As discussed in the previous subsection, the mass $M$ is specified with precision $\Delta M \approx 1 / M l_{\mathrm{P}}^{2}$. The separation of the state as in Eq. (5) is justified because the d.o.f. associated with the black hole microstates-represented by the thermal atmosphere of the black hole in the semiclassical description-are confined in the region $r \leq r_{\mathrm{z}}{ }^{3}$

When sufficient time is passed after a black hole is formed by collapse, the state of the entire system is given by a superposition of terms of the form in Eq. (5) with different black hole masses and locations. The superposition necessarily arises because of the backreaction of Hawking emission [58,59]. The full unitarity of time evolution is retained only if we keep all these terms. However, including this effect is straightforward, and it provides only minor corrections to entropic considerations. We thus use the form of Eq. (5) in discussing the dynamics of the black hole.

After being equilibrated, a black hole can be viewed as static in a timescale shorter than $t_{\mathrm{H}} \approx M l_{\mathrm{P}}^{2}$, at least semiclassically. How does the black hole state $|\psi(M)\rangle$ look then? For now, we ignore the state $|\phi\rangle$ when discussing the black hole. As we will see in Sec. II C 3, this assumption is not quite justified, except possibly for some very early time, but it serves as a good starting point for discussion. Let us consider that the black hole state is perturbed by excitations, e.g., by an infalling object. Such an object can be well described using modes whose frequency $\omega$ (as measured in the asymptotic region) is sufficiently larger than the Hawking temperature

\footnotetext{
${ }^{3}$ Strictly speaking, $|\phi\rangle$ also depends on $M$, but we suppress this argument because it is not important for our purposes. We will also treat excitations spreading both in the $r \leq r_{\mathrm{z}}$ and $r>r_{\mathrm{z}}$ regions approximately by including them either in $|\psi(M)\rangle$ or $|\phi\rangle$. The precise description of these excitations will require a more elaborate expression, but we believe this is an inessential technical subtlety in addressing our problem.
} 


$$
T_{\mathrm{H}}=\frac{1}{8 \pi M l_{\mathrm{P}}^{2}},
$$

as we will see more explicitly in Sec. III A. We therefore separate out these high frequency modes from the rest and call them the hard modes. Below, we write the condition for the hard modes as $\omega \geq \Delta \gg T_{\mathrm{H}}$, although $\Delta$ is not too large: $\Delta \approx O(10) T_{\mathrm{H}} \approx O(1) / M l_{\mathrm{P}}^{2}$.

We group all the orthonormal black hole microstates (in some basis) into sets in such a way that states in the same set have the same configuration for the hard modes. We can do this approximately, which is enough. By labeling the sets by the energy $E$ carried by the hard modes and their members by $i_{E}$, a black hole microstate can be written as

$$
\begin{aligned}
|\psi(M)\rangle= & \sum_{E} \sum_{i_{E}=1}^{\mathcal{N}(M-E)} c_{E i_{E}}|E\rangle\left|\psi_{i_{E}}(M-E)\right\rangle ; \\
& \sum_{E} \sum_{i_{E}=1}^{\mathcal{N}(M-E)}\left|c_{E i_{E}}\right|^{2}=1 .
\end{aligned}
$$

Here, $|E\rangle$ 's are orthonormal states of the hard modes. $\left|\psi_{i_{E}}(M-E)\right\rangle$ for each $E$ are orthonormal states representing members of the set, and we call the modes associated with these states the soft modes. $\mathcal{N}(M-E)$ is given by Eq. (3), where we have identified $\Delta M$ and $\Delta$; as mentioned at the end of the paragraph containing Eq. (3), this does not cause any error in the statistical limit. Note that the Hilbert spaces for the hard and soft modes, as defined here, do not factorize because of the energy constraint.

In writing Eq. (7), we have assumed that the number of independent states for the hard modes is much smaller than that of the soft modes. We will discuss this in Sec. II C 3; here we merely point out that we only consider states that do not yield a significant backreaction on spacetime, which limits the number of possible hard mode states. ${ }^{4}$ We also assume that both types of modes exist only in the region outside the stretched horizon $r \geq r_{\mathrm{s}}$, where

$$
r_{\mathrm{s}}-2 M l_{\mathrm{P}}^{2} \approx O\left(\frac{l_{\mathrm{s}}^{2}}{M l_{\mathrm{P}}^{2}}\right) .
$$

This is motivated by the fact that the spacetime picture breaks down in the region $r<r_{\mathrm{s}}$ due to stringy effects and that the Bekenstein-Hawking entropy is reproduced by integrating the entropy density of the thermal atmosphere in the region $r_{\mathrm{s}} \leq r \leq r_{\mathrm{z}}$, as we will see below. We note that for $|E\rangle$, we include modes on the stretched horizon, e.g., its vibration modes.

\footnotetext{
${ }^{4}$ This implies that the algebra defined on the space of $|E\rangle$ does not close, the situation that often appears in quantum gravity. Here we treat the space as a simple Hilbert space; for a mathematically more rigorous treatment, see, e.g., Ref. [60].
}

Our central assertion is that it is only the hard modes that semiclassical theory can describe at the full quantum level. Here, by semiclassical theory we mean quantum theory with gravity defined on a curved spacetime background (see Sec. III A for further discussion). This makes sense because the other, soft modes cannot be discriminated in the asymptotic region within a timescale at which the black hole background can be viewed as static. This implies that the black hole state at the semiclassical level is obtained after tracing out these soft modes. This leads to

$$
\begin{aligned}
\rho(M) & =\sum_{E} \sum_{i_{E}=1}^{\mathcal{N}(M-E)}\left|c_{E i_{E}}\right|^{2}|E\rangle\langle E| \\
& \simeq \frac{1}{\sum_{E} \mathcal{N}(M-E)} \sum_{E} \mathcal{N}(M-E)|E\rangle\langle E| \\
& =\frac{1}{\sum_{E} e^{-\frac{E}{T_{\mathrm{H}}}}} \sum_{E} e^{-\frac{E}{T_{\mathrm{H}}}}|E\rangle\langle E| .
\end{aligned}
$$

To go to the second line, we have assumed that the black hole state is generic, i.e., the values of $\left|c_{E i_{E}}\right|^{2}$ are statistically the same, ${ }^{5}$ and in the last expression, we have taken the statistical limit $E \ll M$, which we will denote by equality. The expression in Eq. (9) appears as the standard black hole thermal state describing the region $r_{\mathrm{s}} \leq r \leq r_{\mathrm{z}}$. An important point, however, is that the states $|E\rangle$ are supposed to represent only those of the hard modessemiclassical theory does not allow us to describe the microscopic dynamics of the modes associated with energy differences smaller than $\Delta$. The consistency of this picture will be discussed throughout the paper.

\section{Hawking emission}

In this subsection, we will consider implications of the above picture for Hawking emission and evaporation. The discussion below follows initially that of Refs. [21-23], adjusted to the current context. The arguments toward the end of Secs. II C 2 and in II C 3 are new.

\section{Distribution of microscopic information}

In the present picture, semiclassical theory can describe the microscopic dynamics of only the hard modes - the soft modes can be characterized only as a thermal bath of temperature $T_{\mathrm{H}}$ (without a hard component), with which the hard modes interact. It is, however, not only the hard modes in $r_{\mathrm{s}} \leq r \leq r_{\mathrm{z}}$ that can interact with the soft modes. Some of the modes described by $|\phi\rangle$ in Eq. (5), i.e., the "far modes" in $r>r_{\mathrm{z}}$, can also interact with (a small fraction of) the soft modes.

\footnotetext{
${ }^{5}$ If we consider the microcanonical ensemble of the black hole microstates, then we obtain the expression in the second line directly.
} 
To understand this, let us discuss the spatial distribution of the soft modes represented by $\left|\psi_{i_{E}}(M-E)\right\rangle$. The concept of spatial distribution for the soft modes is meaningful despite the fact that their internal dynamics is not known. It is defined through interactions with the hard and far modes, which we will call the "semiclassical modes." Since the dynamics of the semiclassical modes are described by semiclassical theory, there is a well-defined notion of where these modes are located. The distribution of the soft modes can then be determined by analyzing which of the semiclassical modes they mostly interact with.

The expression in Eq. (9) is consistent with the interpretation that the soft and hard modes form an almost closed system equilibrated at the temperature $T_{\mathrm{H}}$. It is, therefore, reasonable to assume that from the viewpoint of the semiclassical modes, the distribution of the soft modes is given by the thermal entropy density of a system with temperature $T_{\mathrm{H}}$ as measured in the asymptotic region. (The deviation from it due to the lack of the hard component is negligible.) Since the black hole microstates comprise the soft modes of all low-energy species, this implies that the spatial distribution of black hole information is given by the entropy density

$$
s(r)=c N(r) T_{\mathrm{loc}}(r)^{3} ; \quad T_{\mathrm{loc}}(r)=\frac{T_{\mathrm{H}}}{\sqrt{1-\frac{2 M l_{\mathrm{P}}^{2}}{r}}},
$$

where $c$ is a constant of $O(1), T_{\mathrm{loc}}(r)$ is the local temperature measured at $r$, and $N(r)$ is the number of low-energy species existing below $T_{\mathrm{loc}}(r)$. We find that integrating this density over the region $r_{\mathrm{s}} \leq r \leq r_{\mathrm{z}}$ indeed reproduces the Bekenstein-Hawking entropy up to an incalculable $O(1)$ factor

$$
\int_{r_{\mathrm{s}}}^{r_{\mathrm{z}}} s(r) \frac{r^{2} d r d \Omega}{\sqrt{1-\frac{2 M l_{\mathrm{P}}^{2}}{r}}} \approx N\left(r_{\mathrm{s}}\right) \frac{M^{2} l_{\mathrm{P}}^{4}}{l_{\mathrm{s}}^{2}} \approx S_{\mathrm{BH}}(M),
$$

where we have assumed that the change of $N(r)$ is not too rapid as a function of $r$ and used the relation expected in any theory of quantum gravity (see, e.g., Ref. [61]):

$$
l_{\mathrm{P}}^{2} \approx \frac{l_{\mathrm{s}}^{2}}{N\left(r_{\mathrm{s}}\right)} .
$$

Note that if we take the lower limit of the integral in Eq. (11) to be the classical horizon $r_{\mathrm{s}} \rightarrow 2 M l_{\mathrm{P}}^{2}$, then the integral diverges. This is consistent with the fact that the entropy of a black hole is infinite at the classical level.

To elucidate the significance of this result in our context, we go to the tortoise coordinate

$$
r^{*}=r+2 M l_{\mathrm{P}}^{2} \ln \frac{r-2 M l_{\mathrm{P}}^{2}}{2 M l_{\mathrm{P}}^{2}},
$$

in which the region outside the Schwarzschild horizon $r \in$ $\left(2 M l_{\mathrm{P}}^{2}, \infty\right)$ is mapped into $r^{*} \in(-\infty, \infty)$. This coordinate is useful in that the kinetic term of an appropriately redefined field takes the canonical form so that its propagation can be analyzed as in flat space. In this coordinate, the stretched horizon located at $r_{\mathrm{s}}=2 M l_{\mathrm{P}}^{2}+O\left(l_{\mathrm{s}}^{2} / M l_{\mathrm{P}}^{2}\right)$ is at

$$
r_{\mathrm{s}}^{*} \simeq-4 M l_{\mathrm{P}}^{2} \ln \frac{M l_{\mathrm{P}}^{2}}{l_{\mathrm{s}}} \simeq-4 M l_{\mathrm{P}}^{2} \ln \left(M l_{\mathrm{P}}\right),
$$

where we have taken $l_{\mathrm{s}}$ to be not too far from $l_{\mathrm{P}}$. This implies that there is a large distance between the stretched horizon and the potential barrier region around the edge of the zone when measured in $r^{*}: \Delta r^{*} \simeq 4 M l_{\mathrm{P}}^{2} \ln \left(M l_{\mathrm{P}}\right) \gg$ $O\left(M l_{\mathrm{P}}^{2}\right)$ for $\ln \left(M l_{\mathrm{P}}\right) \gg 1$. On the other hand, a localized Hawking quantum is represented by a wave packet with width of $O\left(M l_{\mathrm{P}}^{2}\right)$ in $r^{*}$ since it has an energy of order $T_{\mathrm{H}}=$ $1 / 8 \pi M l_{\mathrm{P}}^{2}$ defined in the asymptotic region.

An important point is that the amount of integrated entropy contained around the edge of the zone is of $O(1)$ :

$$
\int_{\left|r^{*}\right| \lesssim O\left(M l_{\mathrm{P}}^{2}\right)} s\left(r\left(r^{*}\right)\right) \sqrt{1-\frac{2 M l_{\mathrm{P}}^{2}}{r\left(r^{*}\right)}} r^{2}\left(r^{*}\right) d r^{*} d \Omega \approx O(1) .
$$

While this is a negligibly small fraction of the total black hole entropy, of order $l_{\mathrm{P}}^{2} / \mathcal{A}(M) \ll 1$, it has a significant implication for the interpretation of the Hawking emission process. It implies that outgoing field theory modesspecifically, outgoing modes represented by $|\phi\rangle$ in Eq. (5) and located around $r \approx r_{\mathrm{z}}$ at the relevant time-can extract black hole information directly from the soft modes at the edge of the zone, without involving a semiclassical mode deep in the zone. In other words, from the viewpoint of the semiclassical modes, microscopic information about the black hole is delocalized over the entire zone, although the distribution is strongly peaked toward the stretched horizon.

While the microscopic dynamics of this information extraction process cannot be described within semiclassical theory because it involves soft modes, the flow of energy and entropy can be investigated using energy-momentum conservation, thermodynamic considerations, and unitarity. This is done below in Sec. II C 2, resulting in the following picture. A Hawking quantum that can be viewed as a semiclassical mode is emitted at the edge of the zone, where it extracts $O(1)$ information from the soft modes in each emission timescale of $t \approx O\left(M l_{\mathrm{P}}^{2}\right)$. Since Hawking evaporation is a long process, this small rate is enough for all the black hole information to be returned to ambient space in the lifetime of the black hole $\tau_{\mathrm{BH}} \approx O\left(M^{3} l_{\mathrm{P}}^{4}\right)$. 


\section{Information transfer}

Let us now amplify the discussion above using a qubit model. Take the black hole state in Eq. (7). We focus on the terms with the lowest $E$

$$
|\psi(M)\rangle \approx \sum_{i=1}^{\mathcal{N}(M)} c_{i}\left|E_{0}\right\rangle\left|\psi_{i}(M)\right\rangle
$$

because for $\Delta \gg T_{\mathrm{H}}$ they statistically dominate the process. Here, $E_{0}=0$ is the lowest value of $E$, and $\Delta$ is the energy separating the hard and soft modes. In the timescale of $O\left(M l_{\mathrm{P}}^{2}\right)$, this state changes by emitting a Hawking quantum. ${ }^{6}$ Suppose that the black hole releases one qubit of information through Hawking emission. The energy of the emitted quantum is then $\Delta M \simeq(\ln 2) / 8 \pi M l_{\mathrm{P}}^{2}$ so that $\mathcal{N}(M-\Delta M)=\mathcal{N}(M) / 2$. We can model this process by saying that the emitted Hawking quantum is in states $\left|r_{1}\right\rangle$ and $\left|r_{2}\right\rangle$ if the index for the soft mode states $i$ is odd and even, respectively. ${ }^{7}$ Because of energy-momentum conservation, the process is accompanied by the creation of an ingoing negative energy excitation, which we denote by a star; namely, $\left|\psi_{i}^{*}(M)\right\rangle$ represents the states of the soft modes with the negative energy excitation.

One might naively think that this process simply goes as

$$
\left|E_{0}\right\rangle\left|\psi_{i}(M)\right\rangle|\phi\rangle \stackrel{?}{\longrightarrow} \begin{cases}\left|E_{0}\right\rangle\left|\psi_{i}^{*}(M)\right\rangle\left|\phi+r_{1}\right\rangle & \text { if } i \text { is odd } \\ \left|E_{0}\right\rangle\left|\psi_{i}^{*}(M)\right\rangle\left|\phi+r_{2}\right\rangle & \text { if } i \text { is even, }\end{cases}
$$

where $\left|\phi+r_{a}\right\rangle(a=1,2)$ is the state in which the outgoing Hawking quantum in state $\left|r_{a}\right\rangle$ is added to the far state $|\phi\rangle$ around the edge of the zone (with the appropriate time evolution). However, this leads to a problem. Remember that $\left|\psi_{i}^{*}(M)\right\rangle$ have energy $M-\Delta M$, and we expect that they will relax into states of the black hole of the decreased mass $M-\Delta M$ :

$$
\left|\psi_{i}^{*}(M)\right\rangle \rightarrow\left|\psi_{i^{\prime}}(M-\Delta M)\right\rangle
$$

Since $i^{\prime}$ runs only over $i^{\prime}=1, \ldots, \mathcal{N}(M-\Delta M)=$ $\mathcal{N}(M) / 2$, however, such a relaxation cannot occur unitarily. Instead, what happens in the emission process must be like

\footnotetext{
${ }^{6}$ Throughout this paper, we assume that the number of species below $T_{\mathrm{H}}$ is small, $N\left(r_{\mathrm{z}}\right) \approx O(1)$, and we mostly focus on the case with a single species. Including the effect of multiple species is straightforward.

${ }^{7}$ In this model, the qubit that escapes from the black hole is the odd-even direction of $i$ in the space spanned by $\left|\psi_{i}(M)\right\rangle$. This corresponds to the statement that the information leaving the black hole is that associated with the soft mode located around $r \sim r_{\mathrm{z}}$.
}

$\left|E_{0}\right\rangle\left|\psi_{i}(M)\right\rangle|\phi\rangle \rightarrow \begin{cases}\left|E_{0}\right\rangle\left|\psi_{\frac{i+1}{2}}^{*}(M)\right\rangle\left|\phi+r_{1}\right\rangle & \text { if } i \text { is odd } \\ \left|E_{0}\right\rangle\left|\psi_{\frac{i}{2}}^{*}(M)\right\rangle\left|\phi+r_{2}\right\rangle & \text { if } i \text { is even }\end{cases}$

i.e., the index for the soft mode states with the negative energy excitation runs only from 1 to $\mathcal{N}(M) / 2$. This allows for these states to relax unitarily into the unexcited soft mode states with the decreased mass $M-\Delta M$, as in Eq. (18). Note that the process in Eq. (19) itself is also unitary if we consider the whole quantum state, including both the black hole and far regions.

The above analysis says that a negative energy excitation over static black hole states, corresponding to the HartleHawking vacuum [62] at the semiclassical level, carries a negative entropy. Namely, in the existence of a negative energy excitation, the range over which the microstate index $i$ runs is smaller than that without. This shows that the standard relation between entropy and energy $S \sim E$ persists even if these quantities are defined with respect to a static black hole background. Specifically, the excitation of energy $-\Delta M$ carries entropy

$$
\Delta S=-8 \pi M \Delta M l_{\mathrm{P}}^{2}=\frac{-\Delta M}{T_{\mathrm{H}}} .
$$

Since a negative energy excitation does not relax instantaneously, the initial states in Eq. (19) may contain multiple negative energy excitations created by earlier emissions. However, we expect that the relaxation time of a negative energy excitation is not much larger than $O\left(M l_{\mathrm{P}}^{2} \ln \left(M l_{\mathrm{P}}\right)\right)$, the time it takes for an excitation to propagate from the edge of the zone to the stretched horizon and also the time it takes for information to be scrambled $[63,64]$. Thus, the number of negative energy excitations existing at any moment is expected to be $\lesssim O\left(\ln \left(M l_{\mathrm{P}}\right)\right)$, which is exponentially smaller than that of the d.o.f. represented by $i$. This implies that negative energy excitations created earlier lie in regions far from the edge of the zone, and hence their effects on the process of Eq. (19) can be safely ignored. ${ }^{8}$

With the microscopic emission process in Eq. (19), a generic black hole state evolves unitarily as described by Page [8]; in particular, the entanglement between the black hole and the emitted Hawking radiation follows the Page curve. As we have seen, the transfer of information from a black hole occurs through a negative entropy flux in the zone carried by ingoing negative energy excitations on a

\footnotetext{
${ }^{8}$ We may redefine the semiclassical vacuum by including these negative energy-entropy excitations. The resulting vacuum will correspond, very roughly, to the Unruh vacuum [34], and the associated geometry is that of an evaporating black hole, which is well described by the advanced/ingoing Vaidya metric near the horizon [65]. In this picture, the change of the local gravitational field supplies the energy of the outgoing Hawking quanta created around $r \sim r_{\mathrm{z}}$.
} 

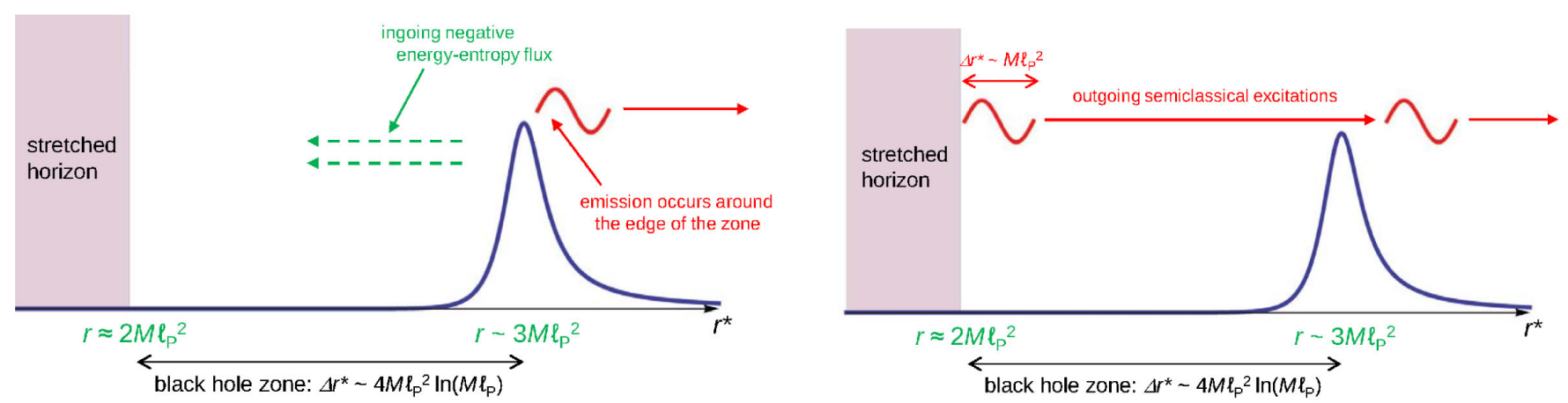

FIG. 1. The information transfer from an evaporating black hole occurs through negative energy-entropy excitations created as a backreaction of Hawking emission occurring around the edge of the zone (left). This can be contrasted with the picture in which outgoing positive energy-entropy excitations carry information from the stretched horizon to the far region (right).

background that can be viewed as static over the timescale of $O\left(M l_{\mathrm{P}}^{2}\right)$. This picture is different from that envisioned in Refs. [18-20], which assumed that information is carried from the stretched horizon to the edge of the zone by outgoing modes described within semiclassical theory; see Fig. 1.

It is important that the transfer of information to semiclassical modes occurs mostly around the edge of the zone, not throughout the zone region. For $\Delta$ sufficiently larger than $T_{\mathrm{H}}$, this condition is guaranteed because production of semiclassical modes in the bulk of the zone is suppressed by

$$
\epsilon \sim\left(\frac{\Delta}{T_{\mathrm{H}}}\right)^{2} e^{-\frac{\Delta}{T_{\mathrm{H}}}}
$$

where the first factor appears because for higher energies larger angular-momentum modes can escape the zone. This might, however, raise the following question. Since the separation energy $\Delta$ between the hard and soft modes is somewhat arbitrary, what happens if we artificially lower $\Delta$ down to $\approx T_{\mathrm{H}}$ ? In this case, "hard modes" defined with respect to the lowered $\Delta$ seem to be produced throughout the zone. However, most of the produced modes cannot propagate to the edge of the zone. The "mean free path" of these modes is of $O\left(M l_{\mathrm{P}}^{2}\right)$ in $r^{*}$ so that only the modes produced around the edge of the zone will escape to ambient space without being reabsorbed by the bath of the soft modes. This is a manifestation of the fact that the modes with frequency $\lesssim T_{\mathrm{H}}$ must be viewed as soft modes, implying that $\Delta$ should be taken sufficiently larger than $T_{\mathrm{H}}$.

We conclude that semiclassical Hawking quanta must be regarded as emitted at the edge of the zone, where information stored in the soft modes-spacetime-is transferred to outgoing semiclassical d.o.f.-Hawking quanta. In fact, it is natural for such special dynamics to occur in this particular region, since this is where the near-horizon, Rindler-like space is "patched" to the asymptotic, Minkowski-like space in the semiclassical picture.
In the timescale of $O\left(M l_{\mathrm{P}}^{2}\right)$, the energy of the emitted Hawking quantum can only be measured with precision of $O\left(1 / M l_{\mathrm{P}}^{2}\right)$. Does this mean that the standard calculation of the gray-body factor is untrustable? It does not. Since the rate of Hawking emission is very small, the change of the black hole mass is very slow. For example, after a very long time of $O\left(M^{2} l_{\mathrm{P}}^{3}\right) \gg M l_{\mathrm{P}}^{2}$, the fractional change of the black hole mass is only of $O\left(1 / M l_{\mathrm{P}}\right) \ll 1$. We then expect that the black hole state keeps taking the form of Eq. (7) with slowly varying $M$ through this long period, and we can apply the standard calculation for this long time, obtaining the result with the error of energy only of $O\left(1 / M^{2} l_{\mathrm{P}}^{3}\right) \ll$ $1 / M l_{\mathrm{P}}$. We find that the standard calculation of the graybody factor, such as that in Ref. [66], can be trusted with precision, parametrically, of $O\left(1 / \tau_{\mathrm{BH}}\right) \approx O\left(1 / M^{3} l_{\mathrm{P}}^{4}\right)$ down to zero energy in the spectrum. This situation is quite different from the modes inside the zone, where the modes with frequency $\lesssim 1 / M l_{\mathrm{P}}^{2}$ interact with the thermal bath in the timescale of $O\left(M l_{\mathrm{P}}^{2}\right)$; hence, these modes must be viewed as soft modes, which do not have a structure beyond thermality at the semiclassical level.

\section{Evolution of microscopic entanglement}

The Hawking emission process at the microscopic level in Eq. (19) indicates that a Hawking quantum shortly after the emission is entangled with the soft mode d.o.f. How does this entanglement evolve?

To address this question, we first argue that the dimension of the Hilbert space associated with the hard modes is exponentially smaller than that associated with the soft modes. In particular, the coarse-grained-or thermalentropy associated with the hard modes is given by either

$$
S_{\text {hard }} \approx O(1)\left(\frac{\mathcal{A}(M)}{l_{\mathrm{P}}^{2}}\right)^{p} ; \quad p<1,
$$




$$
S_{\text {hard }}=c \frac{\mathcal{A}(M)}{l_{\mathrm{P}}^{2}} ; \quad c \ll \frac{1}{4} .
$$

Here and below, we suppress the argument $M$ from entropies. In the language of quantum information in holography, this amounts to saying that the dimension of the code subspace [38] erected on a black hole background (and representing the region $r \geq r_{\mathrm{s}}$ ) is exponentially smaller than that of the Hilbert space associated with the background geometry. We strongly suspect that this is indeed the case, as anticipated earlier [4,37], but we may instead take it as an assumption of the framework. Note that in Refs. [21-23], it was argued that different black hole microstates must be viewed as different "microscopic geometries." This corresponds to the statement that soft modes cannot be represented as d.o.f. specifying states within a code subspace in a way that subsystem recovery is possible; only the hard and far modes can be represented in such a manner (unless an effective description having the second boundary is adopted; see Sec. III B). Below, we assume that Eq. (22) or Eq. (23) is true.

Without any dynamics swapping entanglement in the far region, the purifiers of the Hawking quanta emitted earlier keep being the microstates of the black hole. The state of the combined black hole and Hawking radiation system at a given time is then

$$
\begin{aligned}
|\Psi(M)\rangle= & \sum_{E} \sum_{i_{E}=1}^{\mathcal{N}(M-E)} \sum_{a=1}^{S_{\mathrm{rad}}} c_{E i_{E} a}|E\rangle\left|\psi_{i_{E}}(M-E)\right\rangle\left|r_{a}\right\rangle \\
& \sum_{E} \sum_{i_{E}=1}^{\mathcal{N}(M-E)} \sum_{a=1}^{S_{\mathrm{rad}}}\left|c_{E i_{E} a}\right|^{2}=1
\end{aligned}
$$

where $\left|r_{a}\right\rangle$ 's represent orthonormal states for the radiation, and $S_{\text {rad }}$ is its thermal entropy. The density matrix for the hard modes is given by

$$
\begin{aligned}
\rho_{\mathrm{H}}(M) & =\sum_{E}\left(\sum_{i_{E}=1}^{\mathcal{N}(M-E)} \sum_{a=1}^{e^{\mathrm{r}_{\mathrm{rad}}}}\left|c_{E i_{E} a}\right|^{2}\right)|E\rangle\langle E| \\
& \simeq \frac{1}{\sum_{E} e^{-\frac{E}{T_{\mathrm{H}}}}} \sum_{E} e^{-\frac{E}{T_{\mathrm{H}}}}|E\rangle\langle E|,
\end{aligned}
$$

where in the second line, we have used the fact that the size of $\left|c_{E i_{E} a}\right|^{2}$ is statistically given by ${ }^{9}$

$$
\left|c_{E i_{E} a}\right|^{2} \sim \frac{1}{e^{S_{\mathrm{BH}}+S_{\mathrm{rad}}} \sum_{E} e^{-\frac{E}{T_{\mathrm{H}}}}} .
$$

\footnotetext{
${ }^{9} \mathrm{We}$ are not concerned with the logarithmic correction to the black hole entropy arising from the $\Delta M / M$ factor in Eq. (3), so we will identify $\mathcal{N}(M)$ as $e^{S_{\mathrm{BH}}}$ and similarly for other numbers of microstates.
}

This takes the same form as Eq. (9), so that the physics of the hard modes is still described by standard semiclassical theory. The density matrix for the emitted Hawking radiation, at the time the black hole has mass $M$, is

$$
\rho_{\mathrm{R}}(M)=\sum_{a, b=1}^{e^{S_{\mathrm{rad}}}}\left(\sum_{E} \sum_{i_{E}=1}^{\mathcal{N}(M-E)} c_{E i_{E} a} c_{E i_{E} b}^{*}\right)\left|r_{a}\right\rangle\left\langle r_{b}\right| .
$$

For a quantum chaotic dynamics of the black hole, the von Neumann entropy of this density matrix follows the Page curve as the black hole evaporation progresses.

It is instructive to study the structure of tripartite entanglement in Eq. (24) further. This expression tells us that the state of the hard modes, $\rho_{\mathrm{H}}(M)$ in Eq. (25), is purified by the states of the combined system of soft modes and radiation

$$
\begin{gathered}
|\tilde{E}\rangle=\frac{1}{\sqrt{z_{E}}} \sum_{i_{E}=1}^{\mathcal{N}(M-E)} \sum_{a=1}^{e^{S_{\mathrm{rad}}}} c_{E i_{E} a}\left|\psi_{i_{E}}(M-E)\right\rangle\left|r_{a}\right\rangle ; \\
z_{E}=\sum_{i_{E}=1}^{\mathcal{N}(M-E)} \sum_{a=1}^{e_{\mathrm{rad}}}\left|c_{E i_{E} a}\right|^{2} .
\end{gathered}
$$

Suppose that the coarse-grained/thermal entropies of the three sectors satisfy

$$
S_{\text {hard }} \ll S_{\text {soft }} \approx S_{\text {BH }}, S_{\text {rad }},
$$

which is expected to be valid throughout the history of black hole evolution, except possibly in the earliest time when $S_{\text {rad }} \lesssim S_{\text {hard }}$ (if the black hole equilibrates before $S_{\text {rad }}$ becomes larger than $S_{\text {hard }}$ ). Let us now perform the Schmidt decomposition in the space given by the soft mode and radiation states for each $E$ :

$$
|\tilde{E}\rangle=\sum_{i_{E}=1}^{\mathcal{N}_{E}} \gamma_{E i_{E}}\left|\psi_{i_{E}}\right\rangle\left|r_{i_{E}}\right\rangle ; \quad \sum_{i_{E}=1}^{\mathcal{N}_{E}} \gamma_{E i_{E}}^{2}=1,
$$

where

$$
\mathcal{N}_{E}=\min \left\{\mathcal{N}(M-E), e^{S_{\mathrm{rad}}}\right\},
$$

and $\left|\psi_{i_{E}}\right\rangle$ 's without the argument $M-E$ represent Schmidt basis states. In the above expression, we have kept each entropy only at the leading relevant order in expansion in inverse powers of $M l_{\mathrm{P}}$. By construction, the states of the soft modes as well as those of the radiation in this basis are orthonormal for each $E$ :

$$
\left\langle\psi_{i_{E}} \mid \psi_{j_{E}}\right\rangle=\delta_{i_{E} j_{E}}, \quad\left\langle r_{i_{E}} \mid r_{j_{E}}\right\rangle=\delta_{i_{E} j_{E}},
$$

and all the coefficients in Eq. (31) are real and nonnegative, $\gamma_{E i_{E}} \geq 0$. 
In general, soft mode states corresponding to different $E$ 's are orthogonal

$$
\left\langle\psi_{i_{E}} \mid \psi_{j_{E^{\prime}}}\right\rangle=0 \text { for } E \neq E^{\prime},
$$

but the same is not necessarily true for radiation states. However, the inner product of two generic radiation states is suppressed by the large dimension of the radiation Hilbert space:

$$
\left|\left\langle r_{i_{E}} \mid r_{j_{E^{\prime}}}\right\rangle\right| \approx O\left(\frac{1}{e^{\frac{1}{2} S_{\text {rad }}}}\right) \ll 1 \quad \text { for } E \neq E^{\prime} .
$$

Here, motivated by the expectation that the dynamics of the black hole is quantum chaotic, we have assumed that the distributions of $\mathcal{N}_{E}$ states $\left|r_{i_{E}}\right\rangle$ and $\mathcal{N}_{E^{\prime}}$ states $\left|r_{j_{E^{\prime}}}\right\rangle$ are uncorrelated in the radiation Hilbert space of dimension $e^{S_{\text {rad }}}$. This allows us to view that the state of the entire system takes the form

$$
|\Psi(M)\rangle=\sum_{E} \sqrt{z_{E}} \sum_{i_{E}=1}^{\mathcal{N}_{E}} \gamma_{E i_{E}}|E\rangle\left|\psi_{i_{E}}\right\rangle\left|r_{i_{E}}\right\rangle,
$$

with all the $\left|\psi_{i_{E}}\right\rangle$ 's as well as all the $\left|r_{i_{E}}\right\rangle$ 's being (approximately) orthogonal. Here, $z_{E}$ is defined in Eq. (28).

The entanglement structure in Eq. (35) is reminiscent of the GHZ form [29]. To see the significance of this statement, let us trace out the radiation d.o.f. and obtain the reduced density matrix describing the hard and soft modes

$$
\begin{aligned}
\rho_{\mathrm{HS}}(M)= & \operatorname{Tr}_{\mathrm{rad}}|\Psi(M)\rangle\langle\Psi(M)| \\
= & \sum_{E} \sum_{i_{E}=1}^{\mathcal{N}_{E}} z_{E} \gamma_{E i_{E}}^{2}|E\rangle\left|\psi_{i_{E}}\right\rangle\langle E|\left\langle\psi_{i_{E}}\right| \\
& +\sum_{\substack{E, E^{\prime} \\
E \neq E^{\prime}}} \sum_{i_{E}=1}^{\mathcal{N}_{E}} \sum_{i_{i^{\prime}}^{\prime}=1}^{\mathcal{N}_{E^{\prime}}} \sqrt{z_{E} z_{E^{\prime}}} \gamma_{E i_{E}} \gamma_{E^{\prime} i_{E^{\prime}}} \\
& \times O\left(\frac{1}{e^{\frac{1}{2} S_{\mathrm{rad}}}}\right)|E\rangle\left|\psi_{i_{E}}\right\rangle\left\langle E^{\prime}\right|\left\langle\psi_{i_{i^{\prime}}^{\prime}}\right|,
\end{aligned}
$$

where we have assumed generic sizes for the coefficients

$$
\left|c_{E i_{E} a}\right|^{2} \sim \frac{1}{e^{S_{\mathrm{BH}}+S_{\mathrm{rad}}} \sum_{E} e^{-\frac{E}{T_{\mathrm{H}}}}}, \quad \gamma_{E i_{E}}^{2} \sim \frac{1}{\mathcal{N}_{E}}, \quad z_{E} \sim \frac{e^{-\frac{E}{T_{\mathrm{H}}}}}{\sum_{E} e^{-\frac{E}{T_{\mathrm{H}}}}},
$$

and ignored the irrelevant factor of $e^{S_{\text {hard }} \text {. Note that the }}$ phases of the second term in Eq. (36) are random because of random phases from inner products between different radiation states. Similarly, we can trace out the soft modes and obtain the reduced density matrix for the hard modes and the radiation

$$
\begin{aligned}
\rho_{\mathrm{HR}}(M) & =\operatorname{Tr}_{\mathrm{soft}}|\Psi(M)\rangle\langle\Psi(M)| \\
& =\sum_{E} \sum_{i_{E}=1}^{\mathcal{N}_{E}} z_{E} \gamma_{E i_{E}}^{2}|E\rangle\left|r_{i_{E}}\right\rangle\langle E|\left\langle r_{i_{E}}\right| .
\end{aligned}
$$

This takes the diagonal form.

The expressions in Eqs. (36) and (38) indicate that the correlation of the hard modes with either of the soft modes or radiation is (essentially) classical. For Eq. (38), this is obvious, and for Eq. (36), it is due to the extra $e^{-S_{\text {rad }} / 2}$ factor in the second term originating from Eq. (34). It is striking that the hard modes can be purified only when we consider the combined system of the soft modes and the early radiation. This is the case regardless of the relative size between $S_{\text {soft }}$ and $S_{\text {rad }}$, i.e., whether the age of the black hole is younger or older than the Page time.

Incidentally, the correlation between the soft modes and the radiation given by the reduced density matrix

$\rho_{\mathrm{SR}}(M)=\sum_{E} z_{E} \sum_{i_{E}, i_{E}^{\prime}=1}^{\mathcal{N}_{E}} \gamma_{E i_{E}} \gamma_{E i_{E}^{\prime}}\left|\psi_{i_{E}}\right\rangle\left|r_{i_{E}}\right\rangle\left\langle\psi_{i_{E}^{\prime}}\right|\left\langle r_{i_{E}^{\prime}}\right|$

is generally quantum mechanical as required for the unitary evolution of the black hole. This is a feature that makes the entanglement structure of Eq. (35) different from the true GHZ form.

\section{INTERIOR SPACETIME}

In this section, we study what happens to an object falling into an evaporating black hole. We analyze how the interior spacetime manifests itself in the microscopic description of the black hole. We also discuss relations of this picture with the resolution of the cloning paradox.

\section{A. An object falling into a black hole}

We first analyze an object falling into a black hole. Consider a scalar field $\varphi$ of mass $\mu$. In the tortoise coordinates, its action can be written as

$$
\begin{aligned}
& I= \frac{1}{2} \int d t d r^{*} d \theta d \phi\left(1-\frac{2 M l_{\mathrm{P}}^{2}}{r}\right) r^{2} \sin \theta \\
& \times\left[\frac{1}{1-\frac{2 M l_{\mathrm{P}}^{2}}{r}}\left\{\left(\frac{\partial \varphi}{\partial t}\right)^{2}-\left(\frac{\partial \varphi}{\partial r^{*}}\right)^{2}\right\}-\frac{1}{r^{2}}\left(\frac{\partial \varphi}{\partial \theta}\right)^{2}\right. \\
&\left.\quad-\frac{1}{r^{2} \sin ^{2} \theta}\left(\frac{\partial \varphi}{\partial \phi}\right)^{2}-\mu^{2} \varphi^{2}\right]
\end{aligned}
$$

where $\theta$ and $\phi$ are angular coordinates, and $r$ is a function of $r^{*}$ determined by Eq. (13). By rescaling the field and decomposing into spherical harmonics 

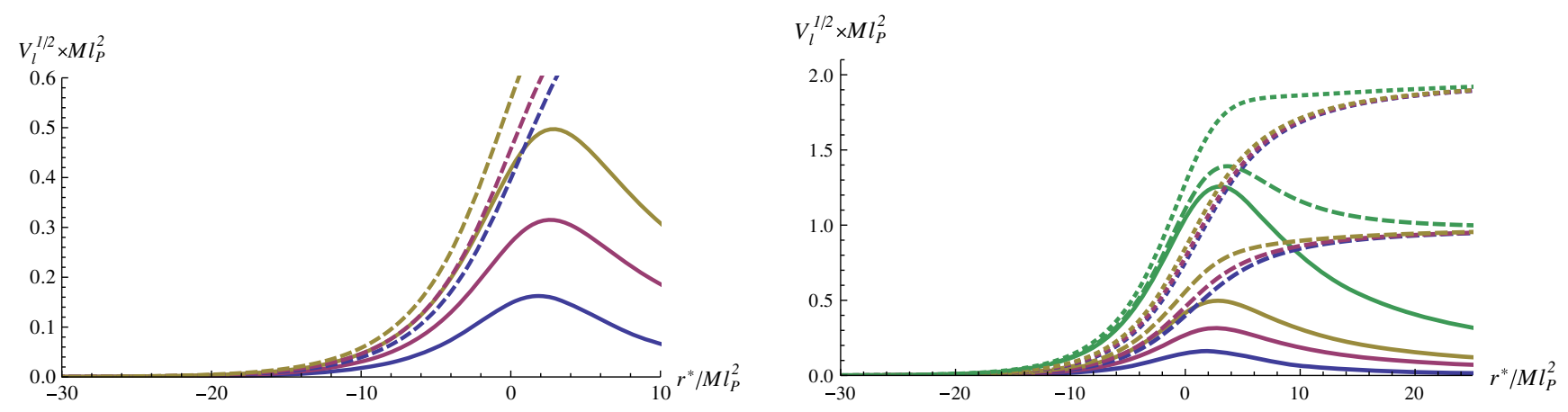

FIG. 2. The potential $\sqrt{V_{l}\left(r^{*}\right)}$ in units of $1 / M l_{\mathrm{P}}^{2}$ plotted as a function of $r_{*} / M l_{\mathrm{P}}^{2}$. In the left panel, solid and dashed lines represent the potential for $\mu=0$ and $1 / M l_{\mathrm{P}}^{2}$, respectively; for each value of $\mu, l=0,1,2$ are plotted (from bottom to top). In the right panel, the cases of $\mu M l_{\mathrm{P}}^{2}=0,1,2$ are plotted on a different scale (solid, dashed, and dotted, respectively), now for $l=0,1,2,5$ for each value of $\mu$ (from bottom to top).

$$
\varphi\left(t, r^{*}, \theta, \phi\right)=\frac{1}{r} \sum_{l, m} \chi_{l m}\left(t, r^{*}\right) Y_{l m}(\theta, \phi),
$$

we obtain

$I=\frac{1}{2} \sum_{l, m} \int d t d r^{*}\left[\left(\frac{\partial \chi_{l m}}{\partial t}\right)^{2}-\left(\frac{\partial \chi_{l m}}{\partial r^{*}}\right)^{2}-V_{l}\left(r^{*}\right) \chi_{l m}^{2}\right]$,

where

$$
V_{l}\left(r^{*}\right)=\left(1-\frac{2 M l_{\mathrm{P}}^{2}}{r}\right)\left(\frac{l(l+1)}{r^{2}}+\frac{2 M l_{\mathrm{P}}^{2}}{r^{3}}+\mu^{2}\right) .
$$

The equation of motion for a mode of frequency $\omega$ is then

$$
-\frac{\partial^{2} \chi_{l m}}{\left(\partial r^{*}\right)^{2}}+V_{l}\left(r^{*}\right) \chi_{l m}=\omega^{2} \chi_{l m}
$$

Note that $\omega$ is a conserved quantity since the action is invariant under translation in $t$. For convenience, we plot $\sqrt{V_{l}\left(r^{*}\right)}$ in Fig. 2 for some small values of $l$ and $\mu$.

Let us first consider the case with $\mu=0$. Suppose we drop a wave packet into a black hole from the outside of the zone, with $r_{\text {init }} \approx O\left(M l_{\mathrm{P}}^{2}\right)>r_{\mathrm{z}}$. We assume that the characteristic width $d$ of the wave packet in the angular directions, which we call the transverse directions, is much smaller than the radius of the black hole: $d \ll R$, where $R=2 M l_{\mathrm{P}}^{2}$. In this case, uncertainty in the transverse momentum is of order $\Delta p_{\perp} \approx 1 / d$, and the frequencies of the modes composing the wave packet have a spread

$$
\Delta \omega \approx \frac{1}{d} \gg \frac{1}{M l_{\mathrm{P}}^{2}}
$$

This implies that the spread of the energy is much larger than the separation energy between the hard and soft modes

$$
\Delta \omega \approx O\left(\frac{R}{d}\right) \Delta \gg \Delta
$$

In terms of angular momentum, the spread is $\Delta L \approx$ $O\left(R \Delta p_{\perp}\right)$, giving

$$
\Delta l \approx O\left(\frac{R}{d}\right) \gg 1
$$

Note that while the peak of the potential $V_{l}\left(r^{*}\right)$ located around $\left|r^{*}\right| \lesssim O\left(M l_{\mathrm{P}}^{2}\right)$ is higher for larger $l$ as $V_{l, \max } \simeq$ $(1 / 27)\left(l^{2} / M^{2} l_{\mathrm{P}}^{4}\right)$, the contributions to the energy in Eq. (46) allow for the wave packet to enter into the zone over the potential barrier.

The situation for $\mu \neq 0$ is similar. In this case, frequencies receive the contribution from the rest mass:

$$
\omega^{2}=\mu^{2}+p^{2}
$$

For $\mu \gtrsim(1 / \sqrt{12}) l / M l_{\mathrm{P}}^{2}$, the effect of gravitation acting on the rest mass makes the barrier disappear; otherwise, the height of the bump is as before, $\Delta V_{l} \simeq(1 / 27)\left(l^{2} / M^{2} l_{\mathrm{P}}^{4}\right)$. The analysis in the case of $\mu=0$ applies essentially with $\omega$ replaced by $p$. In particular, the spreads of various quantities are

$$
\sqrt{\Delta \omega^{2}} \approx \sqrt{\Delta p^{2}} \approx O\left(\frac{R}{d}\right) \Delta \gg \Delta
$$

Note that it is the square of the frequency that is relevant for the dynamics; see Eq. (44). In fact, the term involving the mass in $V_{l}\left(r^{*}\right)$ is negligible compared with $T_{\mathrm{H}}^{2}$ in the region $T_{\text {loc }}(r) \gg \mu$. Therefore, any elementary particle for which $\mu<l_{\mathrm{s}}$ can be regarded as massless near the stretched horizon.

As we discussed in Sec. II, modes with frequencies smaller than $\Delta$ cannot be discriminated at the semiclassical level. The relations in Eqs. (46) and (49), however, show 
that the details of a particle, such as the form of the wave function, can still be described at this level as long as the particle is localized within the length scale $d \ll R$ in the transverse directions. The error coming from neglecting the soft mode contribution to the wave function is suppressed by $d / R$, consistent with the equivalence principle. A similar statement also applies to an object consisting of many particles, whose size is determined not by the spread of the wave function but by interactions between the constituents: As long as its size in the transverse directions is sufficiently smaller than $R$, its dynamics can be well described at the semiclassical level.

This implies that to describe the dynamics of such "small" objects, it is sufficient to consider operators acting on the " $E$ space" in Eq. (24):

$\mathcal{O}|E\rangle\left|\psi_{i_{E}}(M-E)\right\rangle\left|r_{a}\right\rangle=\sum_{E^{\prime}} o_{E E^{\prime}}\left|E^{\prime}\right\rangle\left|\psi_{i_{E}}(M-E)\right\rangle\left|r_{a}\right\rangle$,

where $o_{E E^{\prime}}$ are the elements of a matrix defined in the space spanned by $\{|E\rangle\}$. In particular, small objects in the zone $r_{\mathrm{s}} \leq r \leq r_{\mathrm{z}}$ can be described by the thermal density matrix in Eq. (25) and operators acting on it through

$$
|E\rangle \rightarrow \sum_{E^{\prime}} o_{E E^{\prime}}\left|E^{\prime}\right\rangle
$$

without referring to the state of the soft modes or the early radiation. In fact, the change of the density matrix of the hard modes due to Hawking emission, which occurs through interactions with the soft modes, is sufficiently slow that the description based on these modes can be used for a timescale much longer than $O\left(M l_{\mathrm{P}}^{2}\right.$ ) (where possible changes occurring through a long time period can be treated adiabatically). It is this description that we call semiclassical theory.

\section{B. Emergence of the interior}

What happens when a falling object reaches the stretched horizon? In the viewpoint of a distant observer, the information about the object will be transferred to the excitations of the stretched horizon, which will eventually be resolved into states of the soft modes. However, there is another, coarse-grained description applicable only to certain coarse-grained d.o.f. in a certain limited regime. This leads to the emergence of spacetime inside the horizon. In fact, this is the only sense in which the concept of the black hole interior can come out from the microscopic point of view. In this subsection, we study this issue.

\section{Two-sided description}

Consider a black hole of mass $M$. We label the states for the hard modes $|E\rangle$ in terms of the occupation numbers $n_{\alpha}$ for each mode $\alpha$ :

$$
|E\rangle \rightarrow\left|\left\{n_{\alpha}\right\}\right\rangle .
$$

Here, $\alpha$ collectively denotes the species, frequency, and angular-momentum quantum numbers of the mode. The state of the combined system of the black hole and radiation in Eq. (24) can then be written as

$$
\begin{aligned}
|\Psi(M)\rangle=\sum_{n} & \sum_{i_{n}=1}^{\mathcal{N}\left(M-E_{n}\right)} \sum_{a} c_{n i_{n} a}\left|\left\{n_{\alpha}\right\}\right\rangle\left|\psi_{i_{n}}\left(M-E_{n}\right)\right\rangle\left|r_{a}\right\rangle \\
& \sum_{n} \sum_{i_{n}=1}^{\mathcal{N}\left(M-E_{n}\right)} \sum_{a}\left|c_{n i_{n}}\right|^{2}=1
\end{aligned}
$$

where $n \equiv\left\{n_{\alpha}\right\}$ represents the set of all occupation numbers, and $E_{n}$ is the energy of the state $\left|\left\{n_{\alpha}\right\}\right\rangle$ as measured in the asymptotic region within precision $\Delta$. Important operators of the form of Eq. (50) are annihilation and creation operators

$$
b_{\gamma}=\sqrt{n_{\gamma}}\left|\left\{n_{\alpha}-\delta_{\alpha \gamma}\right\}\right\rangle\left\langle\left\{n_{\alpha}\right\}\right| \otimes \mathbf{1} \otimes \mathbf{1},
$$

$$
b_{\gamma}^{\dagger}=\sqrt{n_{\gamma}+1}\left|\left\{n_{\alpha}+\delta_{\alpha \gamma}\right\}\right\rangle\left\langle\left\{n_{\alpha}\right\}\right| \otimes \mathbf{1} \otimes \mathbf{1},
$$

where 1 represents the fact that the operators do not act on soft mode or radiation states. These comprise operators in the semiclassical theory describing physics in the zone region $r_{\mathrm{s}} \leq r \leq r_{\mathrm{z}}$. As we have seen in Sec. III A, a small object falling toward the horizon can be well described by the configuration of the hard modes, i.e., these operators acting on the black hole state.

As we will see explicitly in Sec. III B 2, we can describe what happens to such a small, falling object without knowing the detailed states of the soft modes or early radiation. We can therefore coarse grain these states as

$$
\begin{aligned}
& \sum_{i_{n}=1}^{\mathcal{N}\left(M-E_{n}\right)} \sum_{a} c_{n i_{n} a}\left|\psi_{i_{n}}\left(M-E_{n}\right)\right\rangle\left|r_{a}\right\rangle \\
& \rightarrow \sqrt{\left.\left.\sum_{i_{n}=1}^{\mathcal{N}\left(M-E_{n}\right)} \sum_{a}\left|c_{n i_{n} a}\right|^{2} \|\left\{n_{\alpha}\right\}\right\rangle\right\rangle .}
\end{aligned}
$$

Here, we have used the same label as the hard mode state to specify the coarse-grained state, which we denote by the double ket symbol, and the coefficient on the right-hand side arises because we have taken $\left.\left.\|\left\{n_{\alpha}\right\}\right\rangle\right\rangle$ to be a normalized state. At the coarse-grained level, the detailed structures of $c_{n i_{n} a}$ 's are not important, so this coefficient can be written as 


$$
\begin{gathered}
\sqrt{\sum_{i_{n}=1}^{\mathcal{N}\left(M-E_{n}\right)} \sum_{a}\left\langle\left|c_{n i_{n} a}\right|^{2}\right\rangle} \approx \sqrt{\frac{\mathcal{N}\left(M-E_{n}\right)}{\sum_{n} \mathcal{N}\left(M-E_{n}\right)}} \\
=\frac{e^{-4 \pi M E_{n} l_{\mathrm{P}}^{2}}}{\sqrt{\sum_{n} e^{-8 \pi M E_{n} l_{\mathrm{P}}^{2}}}}=\frac{e^{-\frac{E_{n}}{2 T_{\mathrm{H}}}}}{\sqrt{\sum_{n} e^{-\frac{E_{n}}{T_{\mathrm{H}}}}}},
\end{gathered}
$$

where

$$
\left\langle\left|c_{n i_{n} a}\right|^{2}\right\rangle \approx \frac{1}{\sum_{n} \sum_{a} \mathcal{N}\left(M-E_{n}\right)}
$$

is the characteristic size of $\left|c_{n i_{n} a}\right|^{2}$ obtained from the normalization condition in Eq. (53). The state in Eq. (53) can then be written as

$$
\left.\| \Psi(M)\rangle\rangle=\frac{1}{\sqrt{\sum_{n} e^{-\frac{E_{n}}{T_{H}}}}} \sum_{n} e^{-\frac{E_{n}}{2 T_{\mathrm{H}}}}\left|\left\{n_{\alpha}\right\}\right\rangle \|\left\{n_{\alpha}\right\}\right\rangle,
$$

which takes the form of the standard thermofield double state in the two-sided black hole picture [34,67], although $\left|\left\{n_{\alpha}\right\}\right\rangle$ 's here represent the states only of the hard modes. ${ }^{10}$

We can now define the "mirror operators" acting on the coarse-grained states [24-26]:

$$
\begin{gathered}
\left.\tilde{b}_{\gamma}=\mathbf{1} \otimes \sqrt{n_{\gamma}} \|\left\{n_{\alpha}-\delta_{\alpha \gamma}\right\}\right\rangle\left\langle\left\langle\left\{n_{\alpha}\right\} \|,\right.\right. \\
\left.\tilde{b}_{\gamma}^{\dagger}=\mathbf{1} \otimes \sqrt{n_{\gamma}+1} \|\left\{n_{\alpha}+\delta_{\alpha \gamma}\right\}\right\rangle\left\langle\left\langle\left\{n_{\alpha}\right\} \|,\right.\right.
\end{gathered}
$$

where the two factors represent operators acting on $\left|\left\{n_{\alpha}\right\}\right\rangle$ (trivially) and $\left.\left.\|\left\{n_{\alpha}\right\}\right\rangle\right\rangle$ in Eq. (59). Note that, as emphasized in Refs. [24-26], these operators cannot be defined in a state-independent manner at the microscopic level; if we want to define operators corresponding to Eqs. (60) and (61) at the microscopic level, then we must do so in a way dependent on the microstate of a black hole. The analysis in Sec. II C indicates that such state-dependent operators must act on both soft modes and early radiation, regardless of the age of the black hole.

In describing a small object falling inside the horizon, however, we need not explicitly consider microscopic operators. This is because as long as we restrict our attention to a certain spacetime region, the dynamics dictating the infalling object can be described unitarily in the Fock space given by (not too many) operators in Eqs. (54), (55), (60), and (61) acting on the state in Eq. (59). In Sec. III B 2, we will specify what the certain spacetime region means.

If Hawking radiation emitted earlier interacts with the environment, it may transfer a part (or all) of its entanglement with the black hole to the environment. The states $\left|r_{a}\right\rangle$ in Eq. (53) must then include the environment as well. In fact, $\left|r_{a}\right\rangle$ can be viewed in general as full states representing the region outside the zone.

What happens if a detector collects a large number of Hawking quanta and then enters into the black hole? Imagine that early Hawking radiation interacts with a detector, leading to different pointer states $\left|d_{I}\right\rangle$. By separating these states from $\left|r_{a}\right\rangle$, the state in Eq. (53) can be written as

$$
|\Psi(M)\rangle=\sum_{n} \sum_{i_{n}=1}^{\mathcal{N}\left(M-E_{n}\right)} \sum_{I} \sum_{a_{I}} c_{n i_{n} I a_{I}}\left|\left\{n_{\alpha}\right\}\right\rangle\left|\psi_{i_{n}}\left(M-E_{n}\right)\right\rangle\left|r_{a_{I}}\right\rangle\left|d_{I}\right\rangle,
$$

where $\sum_{n} \sum_{i_{n}=1}^{\mathcal{N}\left(M-E_{n}\right)} \sum_{I} \sum_{a_{I}}\left|c_{n i_{n} I a_{I}}\right|^{2}=1$. To discuss what the detector finding a particular outcome $I$ will experience later, we may focus on the particular branch of the wave function

$$
\left|\Psi_{I}(M)\right\rangle=\frac{1}{\sqrt{z_{I}}} \sum_{n} \sum_{i_{n}=1}^{\mathcal{N}\left(M-E_{n}\right)} \sum_{a_{I}} c_{n i_{n} I a_{I}}\left|\left\{n_{\alpha}\right\}\right\rangle\left|\psi_{i_{n}}\left(M-E_{n}\right)\right\rangle\left|r_{a_{I}}\right\rangle\left|d_{I}\right\rangle,
$$

\footnotetext{
${ }^{10}$ In this picture, negative energy-entropy excitations of Sec. II $\mathrm{C}$ arising as backreaction of Hawking emission appear as "particles" whose wavelengths are of the order of the black hole horizon radius or an ambiguity in choosing a vacuum at this scale resulting from spacetime curvature. We will ignore this effect, which is not important in discussing physics of a small infalling object.
}

where $z_{I}=\sum_{n} \sum_{i_{n}=1}^{\mathcal{N}\left(M-E_{n}\right)} \sum_{a_{I}}\left|c_{n i_{n} I a_{I}}\right|^{2}$. Generically, this does not affect the physics of the black hole, since the structure of Eq. (63) is the same as that of Eq. (53). However, if the detector is carefully set up, it may be fully correlated with a particular configuration $\left\{n_{\alpha}^{\prime}\right\}$ of the hard modes after the measurement: $c_{n i_{n} I a_{I}} \approx 0$ for $n \neq\left\{n_{\alpha}^{\prime}\right\}$. This seems to mean that when the detector enters the 
horizon, it would hit a "firewall" because the hard modes lack entanglement. This, however, need not be the case.

Since the detector can interact with Hawking quanta only outside the zone, it takes time of order $4 M l_{\mathrm{P}}^{2} \ln \left(M l_{\mathrm{P}}\right)$ for it to reach the stretched horizon. (More generally, it takes time of order $4 M l_{\mathrm{P}}^{2} \ln \left(M l_{\mathrm{P}}\right)$ for the outcome of the measurement to be communicated to the region near the stretched horizon.) Therefore, if the equilibrium timescale between the hard and soft modes is of order

$$
t_{\mathrm{eq}}=4 M l_{\mathrm{P}}^{2} \ln \left(M l_{\mathrm{P}}\right)
$$

or shorter, then the state of the system (without the detector included) takes the form of Eq. (53) with generic $c_{n i_{n} a}=$ $c_{n i_{n} I a_{I}}$ when the detector reaches the stretched horizon. This implies that the detector sees a smooth horizon when it falls into the black hole.

The above analysis suggests that an operation acting only on early Hawking radiation-however complicatedcannot destroy the smoothness of the horizon. This is a consequence of the entanglement structure described in Sec. II C.

\section{Effective theories of the interior}

Suppose that the state of the entire system at a given Schwarzschild time $t=t_{*}$ has a black hole of mass $M$ with a small object in the zone falling toward the horizon. To see what happens to this object, we may adopt the coarse-grained description given above, in which the state is given by

$$
\left.\left.\left.\| \Psi_{0}\right\rangle\right\rangle \propto \prod_{i=1}^{N}\left(\sum_{\gamma} f_{i, \gamma} b_{\gamma}^{\dagger}\right) \| \Psi(M)\right\rangle,
$$

where we have assumed that the object consists of $N$ particles, and $\gamma$ collectively denotes frequency as well as other discrete labels such as those for particle species and angular momenta. The coefficients $f_{i, \gamma}$ are the weights needed to produce particle $i$ by superposing the creation operators $b_{\gamma}^{\dagger}$, and the coarse-grained black hole vacuum state $\| \Psi(M)\rangle\rangle$ is given by Eq. (59). Note that we can always discriminate constituents of a semiclassical object from the thermal atmosphere since they modulate the thermal density matrix of Eq. (25) with energies larger than the energy spread of the black hole vacuum $\Delta E \approx 1 / M l_{\mathrm{P}}^{2}$.

The question is as follows: What does this object experience when it enters the horizon? To answer this question, evolving the state in Schwarzschild time $t$ is of no use. In such a description, the object hits the stretched horizon and is converted into excitations on the stretched horizon, which is then resolved into soft modes due to intrinsically stringy dynamics. In the context of holography, this implies that the boundary time evolution cannot be used to provide the answer since it corresponds to evolution in Schwarzschild time [28]. To address the question, we need to "evolve" the state in a way related to the proper time seen by the object.

Since the coarse-grained black hole vacuum state $\| \Psi(M)\rangle\rangle$ takes the standard thermofield double form, the Fock space built on it can be viewed as representing excitations on a background of the two-sided black hole of mass $M$. The question above, therefore, can be answered by evolving $\left.\left.\| \Psi_{0}\right\rangle\right\rangle$ in Eq. (65) with respect to time $v$ in the non-null Kruskal-Szekeres coordinates:

$$
\left\{\begin{array} { l } 
{ u = \frac { 1 } { 2 } ( - U + V ) , } \\
{ v = \frac { 1 } { 2 } ( U + V ) , }
\end{array} \quad \left\{\begin{array}{l}
U=-R e^{-\tau}, \\
V=R e^{\tau},
\end{array}\right.\right.
$$

where $R$ and $\tau$ are given for $r>2 M l_{\mathrm{P}}^{2}$ by

$$
R=M l_{\mathrm{P}}^{2} \sqrt{\frac{r}{2 M l_{\mathrm{P}}^{2}}-1} e^{\frac{r}{4 M l_{\mathrm{P}}^{2}}}, \quad \tau=\frac{t-t_{*}}{4 M l_{\mathrm{P}}^{2}} .
$$

Specifically, the original annihilation and creation operators $b_{\gamma}, b_{\gamma}^{\dagger}, \tilde{b}_{\gamma}$, and $\tilde{b}_{\gamma}^{\dagger}$ can be related with the new annihilation and creation operators by

$$
\begin{aligned}
& a_{\xi}=\sum_{\gamma}\left(\alpha_{\xi \gamma} b_{\gamma}+\beta_{\xi \gamma} b_{\gamma}^{\dagger}+\zeta_{\xi \gamma} \tilde{b}_{\gamma}+\eta_{\xi \gamma} \tilde{b}_{\gamma}^{\dagger}\right), \\
& a_{\xi}^{\dagger}=\sum_{\gamma}\left(\beta_{\xi \gamma}^{*} b_{\gamma}+\alpha_{\xi \gamma}^{*} b_{\gamma}^{\dagger}+\eta_{\xi \gamma}^{*} \tilde{b}_{\gamma}+\zeta_{\xi \gamma}^{*} \tilde{b}_{\gamma}^{\dagger}\right),
\end{aligned}
$$

where $\xi$ is the label for modes in which the frequency $\omega$ with respect to $t$ is replaced by the frequency $\Omega$ with respect to $v$, i.e., $\xi=\{\Omega, l, m, \ldots\}$, and $\alpha_{\xi \gamma}, \beta_{\xi \gamma}, \zeta_{\xi \gamma}$, and $\eta_{\xi \gamma}$ are the Bogoliubov coefficients calculable using the standard quantum field theory method. The time evolution operator in $v$ is then given by

$$
H_{v}=\sum_{\xi} \Omega a_{\xi}^{\dagger} a_{\xi}+H_{\mathrm{int}}\left(a_{\xi}, a_{\xi}^{\dagger}\right) .
$$

The resulting physics is that of a smooth horizon with interior spacetime.

We stress that the "thermal radiation" in Eq. (25) obtained by tracing out soft (and far) modes is very different from "real radiation" emitted from normal matter, e.g., a piece of coal, which does not admit a similar construction. $^{11}$ In the case of the black hole thermal atmosphere, the form of the density matrix - or temperature-is universal throughout the species, reflecting the fact that its thermal nature arises from entanglement between the hard and soft modes for each species. On the other hand, in the case of radiation from normal matter, the structure of the radiation depends on dynamics. For example, depending on couplings between the constituents of matter and radiation, it is possible that some species (e.g., photons) are radiated but not others (e.g., neutrinos).

\footnotetext{
${ }^{11}$ I thank Raphael Bousso for asking a question that has led me to make the comment here.
} 
The structure of entanglement also depends on the system: Different configurations of radiation are purified by different microscopic configurations of matter. This implies that hard/far-or semiclassical—modes comprising the radiation are purified by other semiclassical modes; in the language of holography, the purifier of radiation states can be found in code subspace d.o.f. that allow for subsystem recovery. The universality discussed here is an important ingredient for the purifier to be interpreted as comprising spacetime, which occurs for the black hole thermal atmosphere and Unruh radiation (see Sec. IV B). ${ }^{12}$

We also emphasize that because of an extremely large boost between distant and infalling reference frames, the evolution in the black hole interior generated by Eq. (70) occurs "instantaneously" from the viewpoint of a distant frame, i.e., within a cutoff time as measured locally at $r \approx r_{\mathrm{s}}$ (within $\approx M l_{\mathrm{P}}^{2}$ in Schwarzschild time). This implies that it is not possible to manipulate soft and far (radiation) modes to affect a mirror state - the state associated with a given hard mode state $\left|\left\{n_{\alpha}\right\}\right\rangle$ in Eq. (53)—within the timescale relevant for the effective description. This provides a further justification for the coarse graining in Eq. (56) and is a key to understand the apparent uniqueness of the infalling vacuum, despite the existence of exponentially many black hole microstates.

There is an important restriction on the applicability of the effective description discussed above. Since $\left|\left\{n_{\alpha}\right\}\right\rangle$ represents states of the hard modes, i.e., the semiclassical modes in the zone $r_{\mathrm{s}} \leq r \leq r_{\mathrm{z}}$, at $\left.\left.t=t_{*}, \|\left\{n_{\alpha}\right\}\right\rangle\right\rangle$ represents states of their mirror modes, i.e., the semiclassical modes in the corresponding mirror region in the second exterior of the two-sided black hole, at the time when the vacuum state takes the thermofield double form Eq. (59) (which we also denote by $t_{*}$ ). The effective description obtained by the coarse graining, therefore, is well defined only in the domain of dependence $D_{\mathrm{z}}$ of the union of the zone and its mirror regions at $t=t_{*}$ in the two-sided description. This implies that a given effective theory defined by $\left.\left.\| \Psi_{0}\right\rangle\right\rangle$ and $H_{v}$ may describe only a part of the history of a falling object.

To illustrate this point, we have depicted in Fig. 3 the trajectories of an object released from $r=4 M l_{\mathrm{P}}^{2}$ at $\left(t-t_{*}\right) / M l_{\mathrm{P}}^{2}=-8,-10,-13,-20$ in the $u-v$ plane (from right to left). The unfilled triangle is the spacetime region in $v \geq 0$ that can be described by this effective theory, which is determined by the location of the edge of the zone at

\footnotetext{
${ }^{12}$ This reveals an intriguing relation between ultraviolet and infrared physics: In order to have spacetime behind the horizon, dynamics at the stretched horizon-i.e., at the string scale $1 / l_{\mathrm{s}}-$ must be chaotic across all low-energy species. Specifically, it redistributes the energy of matter falling into the stretched horizon universally among the species. This provides nontrivial information about the dynamics at the scale $1 / l_{\mathrm{s}}$. In particular, it must not have a structure preventing the universal redistribution, such as an exact global symmetry.
}

$t=t_{*}:(u, v) \simeq\left(1.5 M l_{\mathrm{P}}^{2}, 0\right)$. Note that light rays travel at $45^{\circ}$ in the $u-v$ plane since

$d s^{2}=\frac{32 M l_{\mathrm{P}}^{2}}{r(u, v)} e^{-\frac{r(u, v)}{2 M l_{\mathrm{P}}^{2}}}\left(-d v^{2}+d u^{2}\right)+r(u, v)^{2} d \Omega^{2}$.

We find that the trajectory of the object released at $t=$ $t_{*}-13 M l_{\mathrm{P}}^{2}$ can be described until it hits the singularity, ${ }^{13}$ while other trajectories cannot. To fully describe other trajectories, we need to erect different effective theories building on states at different times. For example, to describe the trajectory of the object released from $r=$ $4 M l_{\mathrm{P}}^{2}$ at $t=t_{*}-8 M l_{\mathrm{P}}^{2}$, we can build an effective theory on the state at $t=t_{*}+5 M l_{\mathrm{P}}^{2}$,

$$
|\Psi\rangle=e^{-i H\left(5 M l_{\mathrm{P}}^{2}\right)}\left|\Psi\left(t=t_{*}\right)\right\rangle,
$$

where $H$ is the time evolution operator in Schwarzschild time $t$.

The existence of a consistent semiclassical description based on the $v$ evolution implies that there is a subsector in the original microscopic theory in which the physics perceived by an object after it crosses the horizon can be described unitarily until it hits the singularity or leaves the spacetime region given by the effective theory. Note that we can always find an effective theory describing the full history of an object. As can be seen from Fig. 3, if we erect an effective theory sufficiently early, the object leaves $D_{z}$ in the positive $u$ direction before it hits the singularity. On the other hand, if the time to erect the effective theory is late, then the object leaves the region in the negative $u$ direction before reaching the singularity. Since the singularity is always located in the spacetime region described by an effective theory, continuity tells us that we can choose a time to erect the effective theory such that the full trajectory of the object is described until it hits the singularity.

So far, we have only considered the infrared cutoff of the effective description provided by the end of the zone: $r \lesssim r_{\mathrm{z}}$ at $t=t_{*}$. However, it is also important to consider the ultraviolet cutoff given by the stretched horizon: $r \geq r_{\mathrm{s}}$ at $t=t_{*} \cdot{ }^{14}$ If there were no such cutoff, as in the case of the classical description, then all the matter that fell into the black hole earlier than $t \simeq t_{*}+4 M l_{\mathrm{P}}^{2} \ln \left(M l_{\mathrm{P}}^{2} / l_{\mathrm{s}}\right)$ (the intersection of the stretched horizon and the future boundary of $D_{z}$ ) would appear in the effective description, with the

\footnotetext{
${ }^{13}$ By an object hitting the singularity, we mean the object entering the region near $r=0$ in which the semiclassical description of gravity breaks down, specifically the region in which a curvature invariant exceeds the string scale, $r \lesssim\left(l_{\mathrm{s}}^{2} l_{\mathrm{P}}^{2} M\right)^{1 / 3}$.

${ }^{14}$ Note that in the effective two-sided description, this excludes the region whose proper distance from the bifurcation surface (at $t=t_{*}$ ) is smaller than the string length, i.e., the union of the region between the mathematical and stretched horizons $2 M l_{\mathrm{P}}^{2} \leq$ $r<r_{\mathrm{s}}$ and its corresponding mirror region in the second exterior.
} 


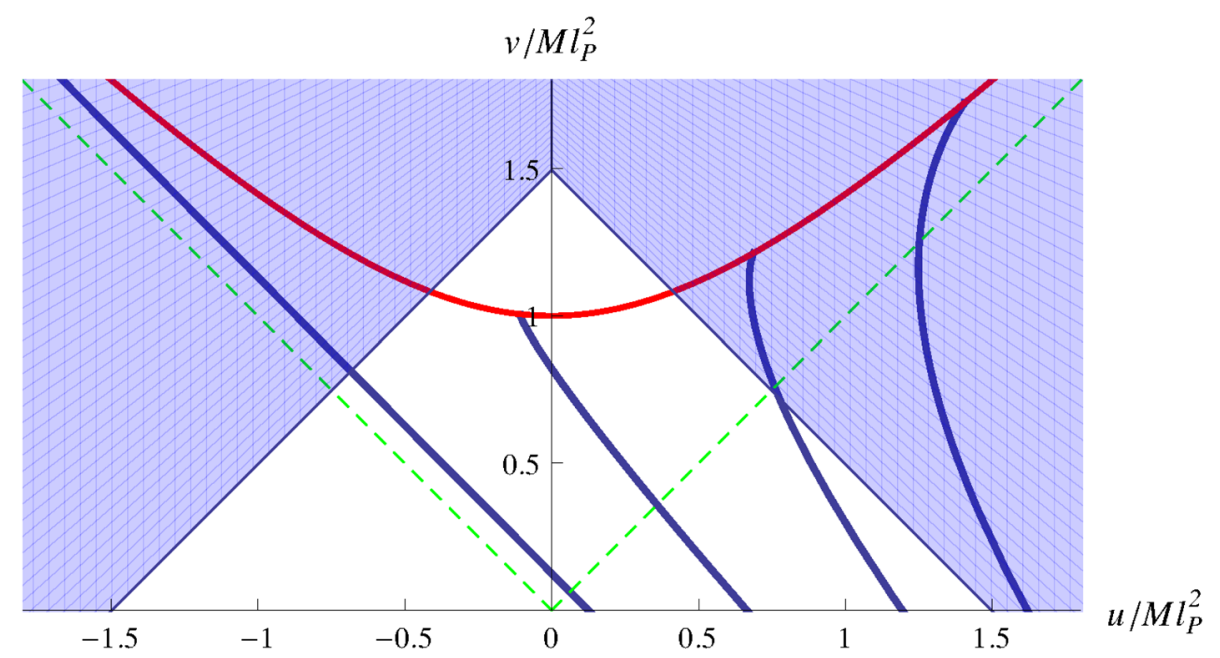

FIG. 3. Trajectories of an object released from $r=4 M l_{\mathrm{P}}^{2}$ at $\left(t-t_{*}\right) / M l_{\mathrm{P}}^{2}=-8,-10,-13,-20$ depicted in the non-null KruskalSzekeres coordinates $(u, v)$ (solid dark-blue lines, from right to left). The unfilled triangle-shaped region represents the positive $v$ part of the spacetime described by the effective theory of the interior built on the full microstate at $t=t_{*}$. The solid red line and dashed green lines represent the singularity $v=\sqrt{u^{2}+M^{2} l_{\mathrm{P}}^{2}}$ and the horizon $v=|u|$, respectively.

trajectories of all the objects that fell earlier than $\approx t_{*}$ concentrated near $v=-u$. This would cause large backreaction on the spacetime, destroying the validity of the effective description. The existence of the ultraviolet cutoff, however, saves the picture.

To see the implications of the ultraviolet cutoff, let us consider an effective theory erected at $t=t_{*}$ and a process in which a falling object sends a null signal in the positive $u$ direction just after passing the horizon. Suppose that the object is at $r=r_{\mathrm{s}}$ at $t=t_{*}$, the location closest to the horizon with the ultraviolet cutoff. The signal then leaves $D_{\mathrm{z}}$ of this effective theory toward positive $u$, but the same signal may also appear in another effective theory erected later at $t=t_{*}+\Delta t$. In order for this to happen, the signal must be sent when

$$
U<R_{\mathrm{Z}} e^{-\frac{t_{*}+\Delta t}{4 M l_{\mathrm{P}}^{2}}}
$$

where $U$ is given by Eq. (66) and

$$
R_{\mathrm{Z}}=M l_{\mathrm{P}}^{2} \sqrt{\frac{r_{\mathrm{Z}}}{2 M l_{\mathrm{P}}^{2}}-1} e^{\frac{r_{\mathrm{z}}}{4 M l_{\mathrm{P}}^{2}}} \simeq 1.5 M l_{\mathrm{P}}^{2} .
$$

Now, in order for the object to send a nontrivial signal, a proper time of order $l_{\mathrm{P}}$ must elapse after it passes the horizon. This is achieved at the smallest $U$ if the object is dropped at $r=r_{\mathrm{s}}\left(\right.$ at $\left.t=t_{*}\right)$ with zero initial velocity. However, this still requires

$$
U>O\left(l_{\mathrm{P}}\right) e^{-\frac{t_{*}}{4 M l_{\mathrm{P}}^{2}}}
$$

for the signal to be sent, where we have taken $l_{\mathrm{P}} \approx l_{\mathrm{S}}$ anticipating the level of precision in the final result. By requiring consistency between Eqs. (73) and (75), we obtain

$$
\Delta t<4 M l_{\mathrm{P}}^{2}\left[\ln \left(M l_{\mathrm{P}}\right)+O(1)\right] .
$$

This implies that the effective theory erected at $t$ cannot receive any signal sent before $\approx t-4 M l_{\mathrm{P}}^{2} \ln \left(M l_{\mathrm{P}}\right)$ inside the horizon.

This strongly suggests that the effective theory erected at $t=t_{*}$ should not include an object that has reached the stretched horizon at

$$
t<t_{*}-4 M l_{\mathrm{P}}^{2}\left[\ln \left(M l_{\mathrm{P}}\right)+O(1)\right] .
$$

Note that in the distant description, an object that has reached the stretched horizon before $t_{*}$ appears as excitations of the stretched horizon modes at $t=t_{*}$. The existence of these modes, together with the fact that an excitation of a hard mode can always be discriminated from the thermal atmosphere, allows us to avoid the frozen vacuum argument in Ref. [68]. It is then natural to associate the cutoff of Eq. (77) with the fact that excitations of the stretched horizon modes are eventually dissipated into soft modes. Specifically, the excitations of the stretched horizon modes caused by an object reached at the stretched horizon before $t_{*}-4 M l_{\mathrm{P}}^{2} \ln \left(M l_{\mathrm{P}}\right)$ have already resolved into the soft modes by the time the effective theory is erected at $t_{*}$. This implies that the timescale for excitations of the stretched horizon to relax into soft modes is

$$
t_{\text {rel }}=4 M l_{\mathrm{P}}^{2} \ln \left(M l_{\mathrm{P}}\right),
$$


up to terms that are not enhanced by $\ln \left(M l_{\mathrm{P}}\right)$. The agreement between this timescale and that in Eq. (64) is suggestive.

Incidentally, the condition of Eq. (77) is identical to the condition that an object must enter $D_{\mathrm{z}}$ of an effective theory before hitting the stretched horizon in order for it to be described by the effective theory. This coincidence is comfortable and strengthens our confidence in the validity of the cutoff given in Eq. (77). With this cutoff, a small object released from $r=r_{0}$ at $t=t_{0}$ with $r_{0}-2 M l_{\mathrm{P}}^{2} \approx O\left(M l_{\mathrm{P}}^{2}\right)$ is included in the effective theory erected at $t_{*}$ only if

$$
t_{0}>t_{*}-8 M l_{\mathrm{P}}^{2}\left[\ln \left(M l_{\mathrm{P}}\right)+O(1)\right] .
$$

This implies that only the object that is dropped at sufficiently late time appears in the effective description. This makes it clear that the issue of large backreaction is avoided.

The discussion above implies that to describe the interior of a black hole "throughout its history," one needs to use multiple effective theories erected at different times (which are generally not mutually independent). The picture of the classical interior spacetime in general relativity emerges only after "patching" descriptions given by these effective theories; see Fig. 4 for a schematic depiction. This is how the picture of complementarity [17] is realized in the present framework. A similar idea was discussed in the context of multiverse cosmology in Ref. [69].

\section{Resolution of the cloning paradox}

A potential issue in a theory in which a black hole evolves unitarily is that of cloning of quantum information

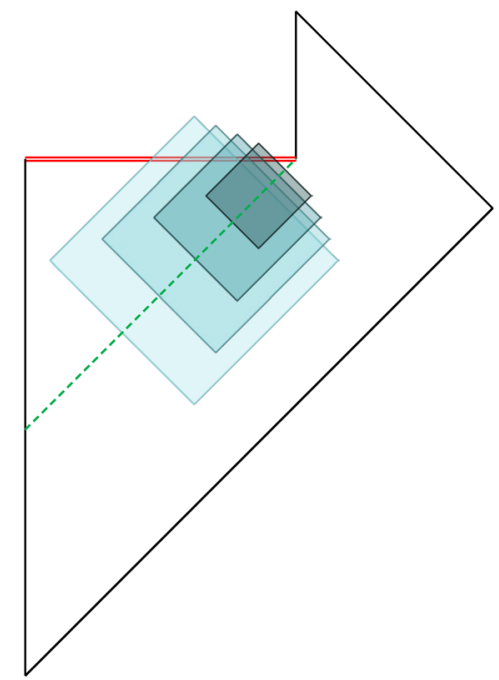

FIG. 4. A series of effective theories erected at different times (depicted by diamonds) covering the interior spacetime. The double red line and the dashed green line represent the singularity and the horizon, respectively. The figure is only a sketch; in particular, the second exterior and the white hole region in each effective theory do not belong to the original spacetime.
$[14,15]$. Suppose that an observer falling into a black hole sends some quantum information along the horizon right after he/she passes the horizon. Suppose also that another observer hovering outside the horizon decodes this information from Hawking radiation (which is possible if the black hole evolution is unitary) and then jumps into the black hole afterward. Now, if the second observer can also receive the signal directly from the first observer after passing the horizon, then it would mean that the second observer has obtained two copies of the same quantum information, which is prohibited by linearity of quantum mechanics [16].

It is often said that this problem is avoided because no one can operationally obtain two copies of information, either because of the time it takes for a black hole to process and send back information $[15,63]$ or an exponentially long time needed for an observer to decode information from Hawking radiation [53]. The framework discussed here, however, provides an arguably simpler solution: There is no duplicate information in any single description, regardless of whether it can be operationally possessed by an observer or not. In this section, we describe this picture. A similar idea has also been discussed in Ref. [70], although the detailed implementations are different.

First, it is clear that no cloning occurs in the distant description because there is no interior. The issue, therefore, is if any effective description of the interior may contain duplicate information. We now argue that the answer is no. In order for the paradox to occur, there must be an infalling object as well as radiation from the black hole that contains the same information. In the effective description, however, there is no Hawking radiation emitted from the edge of the zone since the region outside the zone is not contained in the region described by the effective theory. The thermal atmosphere of the black hole is also absent in this description since the soft modes are already coarse grained to give the semiclassical modes in the mirror zone region. There is simply no way to have information in radiation-or, in fact, radiation itself-in the effective theory describing the interior.

This only leaves the following possibility for information duplication. A detector located in the zone retrieves information about a fallen object and converts it into information in semiclassical d.o.f. before an effective theory describing the falling object in the interior is erected, making both the object and converted information appear in the effective theory. This is, however, impossible. As we have seen in Sec. III B 2, an effective theory erected at $t=t_{*}$ does not describe an object that has reached the stretched horizon before entering the spacetime region $D_{\mathrm{z}}$. This implies that an object must reach the stretched horizon when

$$
t \geq t_{*}-4 M l_{\mathrm{P}}^{2}\left[\ln \left(M l_{\mathrm{P}}\right)+O(1)\right] \equiv t_{1}
$$

in order for it to be included in the description. Here, we have kept explicit only the $\ln \left(M l_{\mathrm{P}}\right)$ enhanced piece regarding 
$\ln \left(l_{\mathrm{s}} / l_{\mathrm{P}}\right) \approx O(1)$. On the other hand, the argument below Eq. (77) implies that information about an object fallen after $t_{1}$ stays as excitations of the stretched horizon modes until $t_{*}$, making it impossible for a detector to extract it from the soft modes.

The discussion above is sufficient to argue that no information duplication occurs in any single description. One might, however, be more satisfied if the information about a falling object described by an effective theory can never reappear in the spacetime region $D_{\mathrm{z}}$ of the effective theory even when the system is described in a distant reference frame. Note that in a distant description, a physical detector may mine information from the black hole thermal atmosphere if it is held in the zone for a sufficiently long time; see Sec. IVA. ${ }^{15}$ This stronger condition eliminates the possibility that a distant description finds reappearance of the information within $D_{\mathrm{z}}$ while the effective description does not. Such a discrepancy between the descriptions is not a contradiction because the effective theory involves coarse graining and hence may lead to information loss, precisely as in the usual semiclassical theory of gravity. Nevertheless, it is aesthetically appealing if this discrepancy never occurs. Below we derive a consequence of requiring this aesthetic criterion and see if it is reasonable to expect that it is satisfied.

The strongest restriction from the requirement arises if an effective theory is erected such that the object reaches the stretched horizon at $t=t_{1}$ given in Eq. (80). The requirement that the information about this objects does not reappear in the zone within $D_{\mathrm{z}}$ implies that it must not reappear from the black hole before

$$
t_{2}=t_{*}+4 M l_{\mathrm{P}}^{2}\left[\ln \left(M l_{\mathrm{P}}\right)+O(1)\right]
$$

the time at which the entirety of the outside region $r>r_{\mathrm{s}}$ leaves $D_{\mathrm{z}}$. Since what leaves $D_{\mathrm{z}}$ last is the region near the stretched horizon $r \approx r_{\mathrm{s}}$, this condition is equivalent to saying that the information about the fallen object should not reappear before $t=t_{2}$ so that even a detector located near the stretched horizon may not probe it. Combining with Eq. (80), we can then conclude that the black hole must retain information longer than $t_{2}-t_{1} \approx$ $8 M l_{\mathrm{P}}^{2} \ln \left(M l_{\mathrm{P}}\right)+O\left(M l_{\mathrm{P}}^{2}\right)$.

Summarizing, the requirement of no information recovery in $D_{\mathrm{z}}$ implies that the information retention time $t_{\mathrm{I}}$ of a black hole of mass $M$ must satisfy

$$
t_{\mathrm{I}} \geq 8 M l_{\mathrm{P}}^{2} \ln \left(M l_{\mathrm{P}}\right)
$$

\footnotetext{
${ }^{15}$ A detector click also occurs in the effective description, which is associated with an emission of a particle rather than an absorption of a particle in the thermal bath [71]. This, however, does not allow for extracting information from the vacuum state since the description is already coarse grained and hence is intrinsically semiclassical
}

up to terms that are not enhanced by $\ln \left(M l_{\mathrm{P}}\right)$. An intriguing point is that the coefficient of the log-enhanced term, i.e., 8 , is determined. In terms of the temperature $T_{\mathrm{H}}$ and entropy $S_{\mathrm{BH}}$ of the black hole, this can be written as

$$
t_{\mathrm{I}} \geq \frac{1}{2 \pi T_{\mathrm{H}}} \ln S_{\mathrm{BH}}=\frac{1}{\lambda_{\mathrm{L}, \max }} \ln S_{\mathrm{BH}},
$$

where $\lambda_{\mathrm{L}, \max }$ is the upper bound on a Lyapunov exponent found in Ref. [72], which a black hole is expected to saturate. This expression makes it natural to expect that the condition in Eq. (82) is indeed satisfied, with the inequality saturated up to non-log-enhanced terms.

\section{RINDLER LIMIT}

In this section, we consider the Rindler limit, aiming to clarify some confusion in the literature regarding the relation between Hawking emission and the Unruh effect. We first discuss black hole mining, which directly corresponds to the Unruh effect in the Rindler limit. We then discuss how physics in Minkowski space arises in this limit, especially focusing on the flow of information.

\section{A. Black hole mining}

The energy and entropy of a black hole can be extracted directly by placing a probe material into the zone $[32,33]$. This process called black hole mining can accelerate the extraction of black hole energy and entropy compared with Hawking emission.

An important difference between mining and Hawking emission processes is the energy cost of angular momentum relative to the local temperature of the thermal atmosphere $T_{\text {loc }}(r)$ in Eq. (10). A similar estimate as in Sec. III A tells us that a particle with angular momentum $L^{2}=l(l+1)$ costs the energy, as measured in the asymptotic region, of

$$
\Delta \omega \approx O\left(\frac{l}{r}\right)
$$

so that

$\frac{\Delta \omega}{T_{\mathrm{loc}}(r)} \approx O\left(\frac{l}{T_{\mathrm{H}} r} \sqrt{1-\frac{2 M l_{\mathrm{P}}^{2}}{r}}\right) \approx O\left(l \sqrt{1-\frac{2 M l_{\mathrm{P}}^{2}}{r}}\right)$.

Therefore, modes up to $l \approx O\left(\sqrt{M l_{\mathrm{P}}^{2} / \delta}\right)$ respond to the thermal atmosphere effectively, where we have taken $r=$ $2 M l_{\mathrm{P}}^{2}+\delta\left(\delta \ll O\left(M l_{\mathrm{P}}^{2}\right)\right)$. This implies that we can a priori utilize many modes

$$
\sum_{l=0}^{l_{\max }}(2 l+1) \approx l_{\max }^{2} \approx O\left(\frac{M l_{\mathrm{P}}^{2}}{\delta}\right)
$$


to extract black hole energy and entropy compared with $O(1)$ (mostly the $s$-wave mode) in the case of Hawking emission. At the stretched horizon, $\delta \approx l_{\mathrm{S}}^{2} / M l_{\mathrm{P}}^{2}$, so that this number is enormous, $\approx O\left(\left(M l_{\mathrm{P}}^{2} / l_{\mathrm{s}}\right)^{2}\right)$.

In realistic situations, energy conditions applied to the probe material prevent us from utilizing all the modes in Eq. (86) for $\delta \lesssim O\left(l_{\mathrm{P}}\right)$; specifically, the null energy condition enforces the number of modes that can be used for mining to be of $O\left(M l_{\mathrm{P}}\right)$ or smaller, so that the black hole lifetime cannot be shorter than $O\left(M^{2} l_{\mathrm{P}}^{3}\right)$ [33]. An important point here, however, is that the rate of extracting energy and entropy for each mode is the same as that in Hawking emission-the acceleration of extraction occurs not because of a higher rate per mode but because of an increased number of modes available to the probe immersed into the zone. This justifies the analysis in Sec. III B 3, which examines the reappearance of information near the stretched horizon to constrain the black hole information retention time.

As in the case of Hawking emission, backreaction of mining causes ingoing negative energy-entropy excitations. A difference is that in the case of mining, these excitations are generally localized in the angular directions. It is expected that the excitations are scrambled in the soft modes at a timescale not much larger than $O\left(M l_{\mathrm{P}}^{2} \ln \left(M l_{\mathrm{P}}\right)\right)$.

\section{B. Semiclassical description in Rindler space}

Here we discuss issues associated with the Rindler limit. We mostly focus on how the semiclassical description of Rindler space is related with that of a black hole. In Sec. IV B 3, we extend the comparison beyond the purely semiclassical regime.

\section{Unruh effect}

Rindler space is obtained as the limiting case of Schwarzschild spacetime

$$
M \rightarrow \infty \quad \text { and } \quad l_{\mathrm{P}}, l_{\mathrm{s}}: \text { fixed }
$$

by focusing on the near horizon region $r \rightarrow 2 M l_{\mathrm{P}}^{2}$, such that the combinations

$$
\rho \equiv 2 \sqrt{2 M l_{\mathrm{P}}^{2}\left(r-2 M l_{\mathrm{P}}^{2}\right)} \quad \text { and } \quad \tau \equiv \frac{t}{4 M l_{\mathrm{P}}^{2}}
$$

are kept finite. The metric in this limit is given by the standard Rindler form

$$
d s^{2}=-\rho^{2} d \tau^{2}+d \rho^{2}+r(\rho)^{2} d \Omega^{2} .
$$

There is no direct analogue of Hawking emission in the Rindler limit since the edge of the zone in the original Schwarzschild spacetime is now at spatial infinity. (We implicitly imagine an infrared cutoff $\rho_{\mathrm{IR}} \rightarrow \infty$ so that
$\rho_{\mathrm{IR}} / M l_{\mathrm{P}}^{2}<\infty$.) There is, however, an analogue of the mining process with a physical probe sensing a thermal bath with temperature

$$
T_{\mathrm{loc}}(\rho)=\lim _{M \rightarrow \infty} \frac{T_{\mathrm{H}}}{\sqrt{1-\frac{2 M l_{\mathrm{P}}^{2}}{r}}}=\frac{1}{2 \pi \rho} .
$$

This is the well-known Unruh effect [34-36]. In the black hole case, mining allows us to extract information about a black hole vacuum. (Recall that a black hole background appears as a black hole vacuum at the semiclassical level, although it actually represents a collection of black hole microstates.) On the other hand, we do not expect to extract information about the Minkowski vacuum from Unruh radiation. Is there a fundamental difference between black hole mining and the Unruh effect, beyond the fact that the latter requires the limit of Eqs. (87) and (88) to be taken?

There is not. In order for information about a black hole vacuum to be extracted, more than a half of its entropy must be mined [8]. The entropy of the Minkowski vacuum, however, is infinite

$$
S_{\text {Minkowski }}=\infty,
$$

as can be seen from the fact that it is obtained by taking the $M l_{\mathrm{P}} \rightarrow \infty$ limit of Schwarzschild spacetime (or the $H l_{\mathrm{P}} \rightarrow 0$ limit of de Sitter space, where $H$ is the Hubble parameter). Therefore, no finite size detector can collect more than a half of the entropy in any finite time. The process of quickly recovering newly added information considered in Ref. [63] is not available either because Minkowski space cannot be maximally entangled with any finite system (and because the "scrambling time" of Rindler space is infinite; see Sec. IV B 3). Information about the Minkowski vacuum, thus, cannot be mined using the Unruh effect by any physical detector-detecting Unruh radiation simply corresponds to an infinitesimally early portion of the Page curve. ${ }^{16}$ This is related to the statement that the BondiMetzner-Sachs [73,74] soft charges, representing microstates of the Minkowski vacuum, cannot be measured by experiments in finite time using a finite-size detector, whose results are determined by the conventional $S$ matrix $[75,76]$.

As in the case of mining a young black hole, detecting Unruh radiation generates entanglement between the detector and the modes represented by the thermal bath, i.e., the soft modes, through the creation of localized negative energy-entropy excitations. This can be understood from the fact that in a Minkowski frame, the Unruh effect corresponds to emission of particles from the detector

\footnotetext{
${ }^{16}$ The fact that Rindler space corresponds to an infinitely young black hole implies that the smoothness of Minkowski space cannot by itself be used to argue against the firewall phenomenon discussed in Refs. [18-20].
} 
[71], which generates entanglement between them. The generated entanglement, however, is finite, so it is infinitesimally small compared with the infinite amount of entropy the soft modes have.

\section{Mirror operators}

The description of Rindler space is analogous to that of a black hole in the zone. In particular, semiclassical theory in Rindler space describes microscopic dynamics of only the hard modes, whose locally measured energies are sufficiently larger than $T_{\text {loc }}(\rho)$. Following the notation in Sec. III B 1, we denote the states of these modes by $\left|\left\{n_{\alpha}\right\}\right\rangle$. The other d.o.f. are regarded as the soft modes, which can be described only statistically.

There is, however, a notable difference arising from taking the limit of Eqs. (87) and (88): The state-dependent nature of constructing the "interior space" (the other side of the Rindler horizon) becomes irrelevant. We first note that since there is no early Hawking mode, the mirror space is constructed purely out of the soft modes. This, however, does not by itself eliminate the need of state dependence. A natural microscopic definition of state-independent mirror operators would be

$\tilde{b}_{\gamma}=\mathbf{1} \otimes \sqrt{n_{\gamma}} \sum_{i=1}^{\mathcal{N}\left(M-E_{n}\right)}\left|\psi_{i}\left(M-E_{n_{-}}\right)\right\rangle\left\langle\psi_{i}\left(M-E_{n}\right)\right|$,

$\tilde{b}_{\gamma}^{\dagger}=\mathbf{1} \otimes \sqrt{n_{\gamma}+1} \sum_{i=1}^{\mathcal{N}\left(M-E_{n_{+}}\right)}\left|\psi_{i}\left(M-E_{n_{+}}\right)\right\rangle\left\langle\psi_{i}\left(M-E_{n}\right)\right|$,

where $\left|\psi_{i}\left(M-E_{n}\right)\right\rangle$ are the soft mode states associated with $\left|\left\{n_{\alpha}\right\}\right\rangle$, and

$$
n_{-}=\left\{n_{\alpha}-\delta_{\alpha \gamma}\right\}, \quad n_{+}=\left\{n_{\alpha}+\delta_{\alpha \gamma}\right\} .
$$

Here, the unit matrices in Eqs. (92) and (93) represent the fact that these operators act trivially on the hard modes, and we have kept the "black hole mass" $M$, although it is taken to infinity in the Rindler limit.

A potential problem lies in the creation operators $\tilde{b}_{\gamma}^{\dagger}$. Suppose $M$ were finite. The number of independent soft mode microstates associated with $\left|n_{+}\right\rangle$would then be smaller than that of $|n\rangle$, which prevents us from defining a microscopic operator that maps any state in the space spanned by $\left|\psi_{i}\left(M-E_{n}\right)\right\rangle$ into a state in the space spanned by $\left|\psi_{i}\left(M-E_{n_{+}}\right)\right\rangle$preserving the appropriate normalization. The fractional difference between the number of independent soft mode states associated with $\left|n_{+}\right\rangle$and $|n\rangle$, however, vanishes in the limit $M \rightarrow \infty$ for fixed energies. The state-independent operators in Eqs. (92) and (93) thus serve as valid mirror operators in this limit.

\section{Information retrieval}

We have seen that the Unruh effect in Rindler space does not allow us to extract information about the Minkowski vacuum. Does this mean that information on the other side of the Rindler horizon, representing a half of Minkowski space, can never be retrieved in a Rindler description?

As seen in Sec. IV B 1, a detection of Unruh radiation creates entanglement between the detector and localized negative energy-entropy excitations of the Rindler soft modes. From a Minkowski point of view, this is entanglement between the detector and particles emitted from it. This entanglement can be retrieved in a Rindler description if we decelerate the detector adiabatically. In other words, if we slowly decrease the acceleration characterizing the Rindler description, then the entanglement-information about the other side of the horizon - can be retrieved in Rindler space in a way that it can be described by semiclassical theory. The physical picture is that as the Rindler horizon recedes due to the deceleration, particles emitted earlier from the detector (in a Minkowski point of view) reappear from the horizon, which increases purity of the system that can be described by semiclassical theory in Rindler space.

This can be viewed as an analogue of information retrieval from a black hole. A difference is that since the entropy of the Minkowski vacuum is infinite, Eq. (91), the "scrambling time" of Rindler space is infinite

$$
\tau_{\text {scr }} \approx O\left(\rho \ln S_{\text {Minkowski }}\right) \rightarrow \infty .
$$

In other words, a state having the negative energy-entropy excitations does not relax into a vacuum state in any finite time. This is the reason why the system reappearing from the horizon (particles emitted earlier from the detector) is not thermalized when it is retrieved.

\section{ENTANGLED BLACK HOLES}

In this section, we discuss entangled black holes. As in the earlier sections, we consider Schwarzschild (or small AdS) black holes. We find that their physics is different from that of commonly considered entangled large AdS black holes in a thermal state.

\section{A. A pair of black holes}

We first consider a pair of entangled black holes. We are not concerned about how it is actually formed. It may, for example, be formed by preparing many Einstein-PodolskyRosen pairs and collapsing them into a black hole in each side of the pairs. Throughout, we assume that each black hole has mass $M$ within precision $\Delta M$.

\section{Entanglement structure}

The most general entanglement structure involving the two black holes is 


$$
|\psi(M, M)\rangle=\sum_{E, F} \sum_{i_{E}=1}^{\mathcal{N}(M-E)} \sum_{j_{F}=1}^{\mathcal{N}(M-F)} \sum_{a} c_{E i_{E} F j_{F} a}|E\rangle_{1}\left|\psi_{i_{E}}(M-E)\right\rangle_{1}|F\rangle_{2}\left|\psi_{j_{F}}(M-F)\right\rangle_{2}\left|r_{a}\right\rangle
$$

where $\sum_{E, F} \sum_{i_{E}=1}^{\mathcal{N}(M-E)} \sum_{j_{F}=1}^{\mathcal{N}(M-F)} \sum_{a}\left|c_{E i_{E} F j_{F} a}\right|^{2}=1$. The subscripts 1 and 2 in the states indicate that they are states of the first and second black holes, respectively, and $\left|r_{a}\right\rangle$ represents states outside the zones of the two black holes, including Hawking radiation emitted earlier.

Tracing out the second black hole in Eq. (96), we obtain

$\rho_{1 \mathrm{R}}(M)=\operatorname{Tr}_{2}|\psi(M, M)\rangle\left\langle\psi(M, M)\left|=\sum_{E, E^{\prime}} \sum_{i_{E}=1}^{\mathcal{N}(M-E)} \sum_{i_{E^{\prime}}^{\prime}=1}^{\mathcal{N}\left(M-E^{\prime}\right)} \sum_{a, a^{\prime}} d_{E i_{E} a E^{\prime} i^{\prime} E^{\prime} a^{\prime}}\right| E\right\rangle_{1}\left|\psi_{i_{E}}(M-E)\right\rangle_{1}\left|r_{a}\right\rangle_{1}\left\langle\left. E^{\prime}\right|_{1}\left\langle\psi_{i_{E^{\prime}}^{\prime}}\left(M-E^{\prime}\right)\right|\left\langle r_{a^{\prime}}\right|\right.$,

where

$$
d_{E i_{E} a E^{\prime} i_{E^{\prime}}^{\prime} a^{\prime}}=\sum_{F} \sum_{j_{F}=1}^{\mathcal{N}(M-F)} c_{E i_{E} F j_{F} a} c_{E^{\prime} i^{\prime}{ }^{\prime} F j_{F} a^{\prime}}^{*}
$$

This is different from the state of the combined system of a nonentangled black hole and radiation in Eq. (24), i.e., $\rho_{1 R}(M) \neq|\psi(M)\rangle\langle\psi(M)|$, unless the system associated with the second black hole decouples, $c_{E i_{E} F j_{F} a}=c_{E i_{E} a_{1}}^{(1)} c_{F j_{F} a_{2}}^{(2)}$ with $\left|r_{a}\right\rangle \rightarrow\left|r_{a_{1}}^{(1)}\right\rangle\left|r_{a_{2}}^{(2)}\right\rangle$. Nevertheless, the reduced density matrix for the hard modes obtained from Eq. (97)

$$
\rho_{\mathrm{H}_{1}}(M)=\operatorname{Tr}_{\mathrm{S}_{1} \mathrm{R}} \rho_{1 \mathrm{R}}(M)=\sum_{E} \sum_{i_{E}=1}^{\mathcal{N}(M-E)} \sum_{a} d_{E i_{E} a E i_{E} a}|E\rangle_{11}\left\langle E\left|\simeq \frac{1}{\sum_{E} e^{-\frac{E}{T_{\mathrm{H}}}}} \sum_{E} e^{-\frac{E}{T_{\mathrm{H}}}}\right| E\right\rangle_{11}\langle E|
$$

takes the standard thermal form, as in Eq. (25). Thus, the physics at the semiclassical level is identical between nonentangled and entangled black holes. Obviously, the same applies to the second black hole as well.

Let us now see the correlation between the hard modes of the two black holes. Tracing out the radiation and soft mode states, we obtain

$$
\begin{aligned}
\rho_{\mathrm{H}_{1} \mathrm{H}_{2}}(M, M) & =\operatorname{Tr}_{\mathrm{S}_{1} \mathrm{~S}_{2} \mathrm{R}}|\psi(M, M)\rangle\langle\psi(M, M)| \\
& =\sum_{E, F}\left(\sum_{i_{E}=1}^{\mathcal{N}(M-E)} \sum_{j_{F}=1}^{\mathcal{N}(M-F)} \sum_{a}\left|c_{E i_{E} F j_{F} a}\right|^{2}\right)|E\rangle_{1}|F\rangle_{21}\left\langle\left. E\right|_{2}\langle F|\right. \\
& \simeq \frac{1}{\sum_{E, F} e^{-\frac{E+F}{T_{\mathrm{H}}}}}\left(\sum_{E} e^{-\frac{E}{T_{\mathrm{H}}}}|E\rangle_{11}\langle E|\right) \otimes\left(\sum_{F} e^{-\frac{F}{T_{\mathrm{H}}}}|F\rangle_{2}\langle F|\right),
\end{aligned}
$$

where in the last line we have assumed genericity of the coefficients $c_{E i_{E} F j_{F} a}$. We find that the hard modes of the two black holes are generically not correlated at the semiclassical level. In fact, the lack of quantum entanglement between these modes is rather general. To illustrate it, consider the special case that the hard modes of the two black holes are correlated at the microscopic level: $c_{E i_{E} F j_{F} a} \propto \delta_{E F}$. In this case, the state of the entire system can be written without loss of generality as

$$
|\psi(M, M)\rangle=\sum_{E} \sum_{i_{E}=1}^{\mathcal{N}(M-E)} \sum_{a} c_{E i_{E} a}|E\rangle_{1}\left|\psi_{i_{E}}(M-E)\right\rangle_{1}|E\rangle_{2}\left|\psi_{i_{E}}(M-E)\right\rangle_{2}\left|r_{a}\right\rangle,
$$

where $\sum_{E} \sum_{i_{E}=1}^{\mathcal{N}(M-E)} \sum_{a}\left|c_{E i_{E} a}\right|^{2}=1$. When the entanglement between the black holes and environment is negligible, $c_{E i_{E} a} \approx c_{E i_{E}} c_{a}$, the two black holes are strongly entangled with each other at the microscopic level; for $c_{E i_{E}}$ 's all having a similar size, they are maximally entangled statistically. Despite this, the correlation between the hard modes of the two black holes is given by 


$$
\rho_{\mathrm{H}_{1} \mathrm{H}_{2}}(M, M)=\sum_{E}\left(\sum_{i_{E}=1}^{\mathcal{N}(M-E)} \sum_{a}\left|c_{E i_{E} a}\right|^{2}\right)|E\rangle_{1}|E\rangle_{2}{ }_{1}\left\langle\left. E\right|_{2}\left\langle E\left|\simeq \frac{1}{\sum_{E} e^{-\frac{E}{T_{\mathrm{H}}}}} \sum_{E} e^{-\frac{E}{T_{\mathrm{H}}}}\right| E\right\rangle_{1} \mid E\right\rangle_{2}{ }_{1}\left\langle\left. E\right|_{2}\langle E|,\right.
$$

so that their correlation is entirely classical.

The origin of the classical nature of the correlation between the hard modes is that in our setup, frequencies of the hard modes, collectively denoted by $\omega$, are larger than the resolution of energy

$$
\omega>\Delta M \text {. }
$$

Note that this situation is different from that considered in Refs. [30,31], which discuss entangled large AdS black holes in a thermal state.

\section{Interior spacetime without a wormhole}

The fact that the correlation between the hard modes of two entangled black holes is at most classical implies that two objects dropped into the two black holes cannot meet inside. Namely, there is no wormhole in the sense that objects dropped into different black holes can meet in interior spacetime. Nevertheless, as we will see below, each object smoothly enters the horizon of the black hole to which it is falling.

As in the case of a nonentangled black hole, the fate of a small object dropped, e.g., into the first black hole, can be described by an effective theory erected around the time when the object reaches the stretched horizon. Assuming that the system is in the state of Eq. (96) at the time the effective theory is erected, mirror states in the effective theory are given by

$$
\| E\rangle_{1}=\frac{1}{\sqrt{z_{E}}} \sum_{i_{E}=1}^{\mathcal{N}(M-E)} \sum_{F} \sum_{j_{F}=1}^{\mathcal{N}(M-F)} \sum_{a} c_{E i_{E} F j_{F} a}\left|\psi_{i_{E}}(M-E)\right\rangle_{1}|F\rangle_{2}\left|\psi_{j_{F}}(M-F)\right\rangle_{2}\left|r_{a}\right\rangle,
$$

where $z_{E}=\sum_{i_{E}=1}^{\mathcal{N}(M-E)} \sum_{F} \sum_{j_{F}=1}^{\mathcal{N}(M-F)} \sum_{a}\left|c_{E i_{E} F j_{F} a}\right|^{2}$. The (vacuum) state in the effective theory, therefore, takes the form

$$
\left.\left.\left.\left.\| \psi(M)\rangle\rangle_{1}=\sum_{E} \sqrt{z_{E}}|E\rangle_{1} \| E\right\rangle\right\rangle_{1} \simeq \frac{1}{\sqrt{\sum_{E} e^{-\frac{E}{T_{\mathrm{H}}}}}} \sum_{E} e^{-\frac{E}{2 T_{\mathrm{H}}}}|E\rangle_{1} \| E\right\rangle\right\rangle_{1}
$$

where genericity of the coefficients $c_{E i_{E} F j_{F} a}$ is assumed in the second line. The object falling into the first black hole can be described by operators analogous to those in Eqs. (54), (55), (60), and (61) acting on appropriate factors of Eq. (105). The resulting physics is that of a smooth horizon for the first black hole.

The same is true for an object falling into the second black hole. Its fate is described by an effective theory erected around the time when the object reaches the stretched horizon of the second black hole, now with the identification

$$
\| F\rangle_{2}=\frac{1}{\sqrt{z_{F}}} \sum_{E} \sum_{i_{E}=1}^{\mathcal{N}(M-E)} \sum_{j_{F}=1}^{\mathcal{N}(M-F)} \sum_{a} c_{E i_{E} F j_{F}}|E\rangle_{1}\left|\psi_{i_{E}}(M-E)\right\rangle_{1}\left|\psi_{j_{F}}(M-F)\right\rangle_{2}\left|r_{a}\right\rangle,
$$

where $z_{F}=\sum_{E} \sum_{i_{E}=1}^{\mathcal{N}(M-E)} \sum_{j_{F}=1}^{\mathcal{N}(M-F)} \sum_{a}\left|c_{E i_{E} F j_{F}}\right|^{2}$. This leads to

$$
\left.\left.\left.\| \psi(M)\rangle\rangle_{2}=\sum_{F} \sqrt{z_{F}}|F\rangle_{2} \| F\right\rangle_{2} \simeq \frac{1}{\sqrt{\sum_{F} e^{-\frac{F}{T_{\mathrm{H}}}}}} \sum_{F} e^{-\frac{F}{2 T_{\mathrm{H}}}}|F\rangle_{2} \| F\right\rangle\right\rangle_{2}
$$

The object falling into the second black hole is described by operators analogous to those in Eqs. (54), (55), (60), and (61) acting on this state, which sees smooth spacetime when entering the horizon.

\section{B. More than two black holes}

The analysis described above can be easily generalized to more than two black holes with arbitrary masses. For $n$ black holes with masses $M_{\alpha}(\alpha=1, \ldots, n)$, the general state can be written as 


$$
\left|\psi\left(\left\{M_{\alpha}\right\}\right)\right\rangle=\left(\prod_{\alpha=1}^{n} \sum_{E_{\alpha}} \sum_{i_{E_{\alpha}}=1}^{\mathcal{N}\left(M_{\alpha}-E_{\alpha}\right)}\right) \sum_{a} c_{\left\{E_{\alpha}\right\}\left\{i_{E_{\alpha}}\right\} a}\left(\prod_{\alpha=1}^{n}\left|E_{\alpha}\right\rangle_{\alpha}\left|\psi_{i_{E_{\alpha}}}\left(M_{\alpha}-E_{\alpha}\right)\right\rangle_{\alpha}\right)\left|r_{a}\right\rangle .
$$

We can trace out various components to see the entanglement structure of the state.

A general feature of these systems is that the correlations between the hard modes of different black holes are at most classical. In particular, for generic microscopic entanglement, we find

$$
\begin{aligned}
\rho_{\mathrm{H}}\left(\left\{M_{\alpha}\right\}\right) & =\operatorname{Tr}_{\mathrm{SR}}\left|\psi\left(\left\{M_{\alpha}\right\}\right)\right\rangle\left\langle\psi\left(\left\{M_{\alpha}\right\}\right)\right| \\
& =\sum_{E_{\alpha}}\left\{\left(\prod_{\alpha=1}^{n} \sum_{i_{E_{\alpha}}=1}^{\mathcal{N}\left(M_{\alpha}-E_{\alpha}\right)}\right) \sum_{a}\left|c_{\left\{E_{\alpha}\right\}\left\{i_{E_{\alpha}}\right\} a}\right|^{2}\right\}\left(\prod_{\alpha=1}^{n}|E\rangle_{\alpha \alpha}\langle E|\right) \\
& \simeq \frac{1}{\sum_{E_{\alpha}} e^{-\frac{\Sigma_{\alpha} E_{\alpha}}{T_{\mathrm{H}}}}} \bigotimes_{\alpha=1}^{n}\left(\sum_{E_{\alpha}} e^{-\frac{E_{\alpha}}{T_{\mathrm{H}}}}|E\rangle_{\alpha \alpha}\langle E|\right) .
\end{aligned}
$$

Small semiclassical objects dropped into different black holes, therefore, cannot meet inside. It is also not possible to make the wormhole traversable by connecting hard modes of different black holes through small direct interactions. $^{17}$

\section{CONCLUSIONS AND DISCUSSION}

In this paper, we have analyzed the quantum mechanics of an evaporating black hole. A key ingredient is that semiclassical theory in a distant view (a view based on Schwarzschild time) describes microscopic dynamics of only the hard modes: the d.o.f. that are hard enough to be discriminated within the characteristic timescale of black hole evolution, $t_{\mathrm{H}} \approx M l_{\mathrm{P}}^{2}$. In an equilibrated black hole, these d.o.f. must be entangled with the rest of the black hole d.o.f., which are too soft to be discriminated due to a large redshift caused by the black hole. This is because the differences of the energy between different configurations of the hard modes are compensated by those of the soft modes in a black hole microstate, whose energy is determined with precision of order $1 / t_{\mathrm{H}}$. In fact, this equilibrium between the hard and soft modes is the origin of the thermodynamic nature of a black hole seen at the semiclassical level.

Based on intuition coming from comparing usual thermodynamic entropies of matter with the Bekenstein-Hawking entropy, we expect that the number of the hard mode d.o.f. is much smaller than that of the soft mode d.o.f. This implies that the former is much smaller than both the latter and the number of early Hawking radiation d.o.f.

$$
S_{\text {hard }} \ll S_{\text {soft }}, S_{\text {rad }}
$$

\footnotetext{
${ }^{17}$ We can have standard wormhole phenomena between semiclassical objects and "objects" that are cleverly composed of hard, soft, and radiation d.o.f. such that they appear as semiclassical objects dropped from mirror space.
}

throughout the history of the black hole evolution (except possibly in the very earliest time). Here, $S_{\text {hard }}, S_{\text {soft }}, S_{\text {rad }}$ are the coarse-grained entropies of the hard modes, soft modes, and early radiation, respectively. We have seen that this makes the entanglement between these three types of d.o.f. intrinsically tripartite

$$
|\Psi(M)\rangle \sim \sum_{E} e^{-\frac{E}{2 T_{H}}} \sum_{i_{E}=1}^{\mathcal{N}_{E}} \frac{1}{\sqrt{\mathcal{N}_{E}}}|E\rangle\left|\psi_{i_{E}}\right\rangle\left|r_{i_{E}}\right\rangle,
$$

where $\mathcal{N}_{E}=\min \left\{e^{S_{\text {sot }, E}}, e^{S_{\text {rad }}}\right\}$ with $S_{\text {soft }, E}$ being the coarsegrained entropy of the soft modes associated with $|E\rangle$. This structure resembles that of the GHZ state in that the correlation between the hard and soft modes as well as that between the hard modes and early radiation are classical, although the correlation between the soft modes and early radiation is generally quantum mechanical as required by unitarity. This implies that mirror modes needed for a theory of the interior consist of both soft modes and early radiation.

When viewed from a distance, the physics of an evaporating black hole is not so mysterious after all. Scattering of high-energy particles, or gravitational collapse, forms a bound state with a high density of states-a black hole-which decays into asymptotic Hawking quanta. While microstates of the bound state at an intermediate stage cannot be resolved from the asymptotic region due to a large gravitational redshift, the standard rules of thermodynamics are obeyed $[77,78]$ throughout the process. A mystery arises (only) if we consider the interior spacetime. The origin of the mystery is, again, the large redshift. When viewed from a distance, an observer falling into a black hole is absorbed into the hot, stretched horizon, and yet we expect the existence of a description in which the observer falls smoothly inside the horizon, at least until it approaches the singularity. We have seen that such a description can be obtained in an effective theory erected at a fixed time obtained after coarse graining microscopic d.o.f. that cannot be discriminated within the timescale of 
$t_{\mathrm{H}}$. This effective theory is intrinsically semiclassical-the description of a falling object is unitary only until it hits the singularity (or escapes the spacetime region to which the effective theory is applicable). This suggests that it is meaningless to ask what happens to a fallen object "after it hits the singularity" within the interior picture. The only fundamental description, which can be unitary for an arbitrarily long time, is that in a distant frame. In the context of holography, this corresponds to the holographic, boundary description of the system.

The results presented in this paper provide a picture of how the peculiarities of a black hole emerge in a holographic boundary theory. A black hole in the semiclassical description corresponds to a high density of states having energetically and spatially similar profiles in a code subspace. As a result of this degeneracy, arising from a large gravitational redshift, a vast majority of the d.o.f. associated with these states cannot be represented as those allowing for subsystem recovery. ${ }^{18}$ Only a tiny fraction-the hard modes-allow for such recovery, having direct association with spacetime. The d.o.f. that do not admit subsystem recovery - the soft modes - are indistinguishable within a characteristic timescale for the boundary evolution of these states; discriminating them requires much longer time in which the degeneracy is resolved by evolution involving Hawking evaporation. However, precisely because of this indistinguishability, we can have a new coarse-grained description applicable in the characteristic timescale. Such a description is useful for knowing the fate of a small object falling into the black hole. Because of the large redshift, the applicability of this effective description is limited to a timescale of order the string/cutoff scale (multiplied by a logarithmic factor) locally near the stretched horizon; however, due to an extremely large boost, this corresponds to a macroscopic timescale perceived by the object. The effective description is possible because after the coarse graining, collective excitations of the soft modes (and the

\footnotetext{
${ }^{18}$ In the boundary picture, this occurs because the entanglement structure of a boundary state becomes such that when the boundary is pulled in to the stretched horizon by coarse graining short-range correlations [28], the resulting boundary state in the effective Hilbert space becomes a generic, maximally entropic state. This prevents us from erecting a code subspace with subsystem recovery since the state does not have a resource of "unentanglement" needed to have such a space [79].
}

d.o.f. entangling with them, including early Hawking radiation) appear as the mirror of the hard modes, with the entanglement between the mirror and the original hard modes taking a thermofield double form. This allows for reinterpreting these collective modes as representing d.o.f. that admit subsystem recovery in a code subspace erected on the extended, second boundary, which is isomorphic to the original boundary renormalized down to the edge of the zone, à la Ref. [28]. In the bulk, this gives the two-sided black hole picture applicable within the domain of dependence of the union of the zone and its mirror regions.

The physics of a black hole has a parallel in Minkowski space: an accelerating detector measuring Unruh radiation. As in the case of a young black hole, this introduces entanglement between the detector and radiation d.o.f. From the point of view of an inertial observer, this is entanglement between the detector and particles emitted from it. This entanglement can be retrieved in Rindler space if we decelerate the detector adiabatically. This can be viewed as an analogue of information retrieval from a black hole, although the retrieved information in this case is not thermalized because of the infinite entropy of the Minkowski vacuum.

The discussion described above suggests that we may view the black hole interior as a sort of "compactified (half) Minkowski space." As viewed from the exterior, the finiteness of the system leads to thermalization/scrambling of information retrieved from a black hole. From the viewpoint of the interior, it is reflected in the fact that the description is fundamentally nonunitary, a manifestation of which is the existence of the singularity. It would be interesting to see if other singularities in general relativity could be understood in similar manners.

\section{ACKNOWLEDGMENTS}

I thank Kanato Goto, Masahiro Nozaki, Pratik Rath, Nico Salzetta, Fabio Sanches, and Sean Weinberg for discussion on this and related topics. This work was supported in part by the Department of Energy, Office of Science, Office of High Energy Physics under Contract No. DE-AC02-05CH11231 and Award No. DE-SC0019380, by the National Science Foundation under Grant No. PHY-1521446, and by the Ministry of Education, Culture, Sports, Science, and Technology-Japan under KAKENHI Grant No. 15H05895.
[1] J. D. Bekenstein, Black holes and entropy, Phys. Rev. D 7, 2333 (1973).

[2] S. W. Hawking, Black hole explosions, Nature (London) 248, 30 (1974).
[3] S. W. Hawking, Particle creation by black holes, Commun. Math. Phys. 43, 199 (1975); 46, 206 (1976).

[4] G. 't Hooft, Dimensional reduction in quantum gravity, in Salamfestschrift, edited by A. Ali, J. Ellis, and 
S. Randjbar-Daemi (World Scientific, Singapore, 1994), p. 284.

[5] L. Susskind, The world as a hologram, J. Math. Phys. (N.Y.) 36, 6377 (1995).

[6] R. Bousso, A covariant entropy conjecture, J. High Energy Phys. 07 (1999) 004.

[7] G. 't Hooft, On the quantum structure of a black hole, Nucl. Phys. B256, 727 (1985).

[8] D. N. Page, Information in Black Hole Radiation, Phys. Rev. Lett. 71, 3743 (1993).

[9] N. Arkani-Hamed, L. Motl, A. Nicolis, and C. Vafa, The string landscape, black holes and gravity as the weakest force, J. High Energy Phys. 06 (2007) 060.

[10] J. Maldacena, The large $N$ limit of superconformal field theories and supergravity, Int. J. Theor. Phys. 38, 1113 (1999); Adv. Theor. Math. Phys. 2, 231 (1998).

[11] S. S. Gubser, I. R. Klebanov, and A. M. Polyakov, Gauge theory correlators from noncritical string theory, Phys. Lett. B 428, 105 (1998).

[12] E. Witten, Anti-de Sitter space and holography, Adv. Theor. Math. Phys. 2, 253 (1998).

[13] S. W. Hawking, Breakdown of predictability in gravitational collapse, Phys. Rev. D 14, 2460 (1976).

[14] J. Preskill (unpublished).

[15] L. Susskind and L. Thorlacius, Gedanken experiments involving black holes, Phys. Rev. D 49, 966 (1994).

[16] W. K. Wootters and W. H. Zurek, A single quantum cannot be cloned, Nature (London) 299, 802 (1982).

[17] L. Susskind, L. Thorlacius, and J. Uglum, The stretched horizon and black hole complementarity, Phys. Rev. D 48, 3743 (1993).

[18] A. Almheiri, D. Marolf, J. Polchinski, and J. Sully, Black holes: Complementarity or firewalls?, J. High Energy Phys. 02 (2013) 062.

[19] A. Almheiri, D. Marolf, J. Polchinski, D. Stanford, and J. Sully, An apologia for firewalls, J. High Energy Phys. 09 (2013) 018.

[20] D. Marolf and J. Polchinski, Gauge/Gravity Duality and the Black Hole Interior, Phys. Rev. Lett. 111, 171301 (2013).

[21] Y. Nomura, F. Sanches, and S. J. Weinberg, Black Hole Interior in Quantum Gravity, Phys. Rev. Lett. 114, 201301 (2015).

[22] Y. Nomura, F. Sanches, and S. J. Weinberg, Relativeness in quantum gravity: Limitations and frame dependence of semiclassical descriptions, J. High Energy Phys. 04 (2015) 158.

[23] Y. Nomura and N. Salzetta, Why firewalls need not exist, Phys. Lett. B 761, 62 (2016).

[24] K. Papadodimas and S. Raju, An infalling observer in AdS/CFT, J. High Energy Phys. 10 (2013) 212.

[25] K. Papadodimas and S. Raju, State-dependent bulkboundary maps and black hole complementarity, Phys. Rev. D 89, 086010 (2014).

[26] K. Papadodimas and S. Raju, Remarks on the necessity and implications of state-dependence in the black hole interior, Phys. Rev. D 93, 084049 (2016).

[27] J. Maldacena and L. Susskind, Cool horizons for entangled black holes, Fortschr. Phys. 61, 781 (2013).

[28] Y. Nomura, P. Rath, and N. Salzetta, Pulling the boundary into the bulk, Phys. Rev. D 98, 026010 (2018).
[29] D. M. Greenberger, M. A. Horne, and A. Zeilinger, Going beyond Bell's theorem, in Bell's Theorem, Quantum Theory, and Conceptions of the Universe, edited by M. Kafatos (Kluwer, Dordrecht, 1989), p. 69.

[30] J. M. Maldacena, Eternal black holes in anti-de Sitter, J. High Energy Phys. 04 (2003) 021.

[31] P. Gao, D. L. Jafferis, and A. C. Wall, Traversable wormholes via a double trace deformation, J. High Energy Phys. 12 (2017) 151.

[32] W. G. Unruh and R. M. Wald, Acceleration radiation and generalized second law of thermodynamics, Phys. Rev. D 25, 942 (1982).

[33] A. R. Brown, Tensile Strength and the Mining of Black Holes, Phys. Rev. Lett. 111, 211301 (2013).

[34] W. G. Unruh, Notes on black hole evaporation, Phys. Rev. D 14, 870 (1976).

[35] S. A. Fulling, Nonuniqueness of canonical field quantization in Riemannian space-time, Phys. Rev. D 7, 2850 (1973).

[36] P. C. W. Davies, Scalar particle production in Schwarzschild and Rindler metrics, J. Phys. A 8, 609 (1975).

[37] Y. Nomura and S. J. Weinberg, Entropy of a vacuum: What does the covariant entropy count?, Phys. Rev. D 90, 104003 (2014).

[38] A. Almheiri, X. Dong, and D. Harlow, Bulk locality and quantum error correction in AdS/CFT, J. High Energy Phys. 04 (2015) 163.

[39] S. B. Giddings, Black holes, quantum information, and unitary evolution, Phys. Rev. D 85, 124063 (2012).

[40] S. B. Giddings, Nonviolent nonlocality, Phys. Rev. D 88, 064023 (2013).

[41] S. B. Giddings, Nonviolent information transfer from black holes: A field theory parametrization, Phys. Rev. D 88, 024018 (2013).

[42] Y. Nomura and S. J. Weinberg, Black holes, entropies, and semiclassical spacetime in quantum gravity, J. High Energy Phys. 10 (2014) 185.

[43] W. Israel, Shenanigans at the black hole horizon: Pair creation or Boulware accretion?, arXiv:1504.02419.

[44] S. B. Giddings, Hawking radiation, the Stefan-Boltzmann law, and unitarization, Phys. Lett. B 754, 39 (2016).

[45] S. B. Giddings, Nonviolent unitarization: Basic postulates to soft quantum structure of black holes, J. High Energy Phys. 12 (2017) 047.

[46] E. Verlinde and H. Verlinde, Black hole entanglement and quantum error correction, J. High Energy Phys. 10 (2013) 107.

[47] Y. Nomura and J. Varela, A note on (no) firewalls: The entropy argument, J. High Energy Phys. 07 (2013) 124.

[48] E. Verlinde and H. Verlinde, Passing through the firewall, arXiv:1306.0515.

[49] Y. Nomura, J. Varela, and S. J. Weinberg, Black holes or firewalls: A theory of horizons, Phys. Rev. D 88, 084052 (2013).

[50] E. Verlinde and $H$. Verlinde, Behind the horizon in AdS/CFT, arXiv:1311.1137.

[51] R. Bousso, Complementarity is not enough, Phys. Rev. D 87, 124023 (2013).

[52] T. Jacobson, Boundary unitarity and the black hole information paradox, Int. J. Mod. Phys. D 22, 1342002 (2013). 
[53] D. Harlow and P. Hayden, Quantum computation vs. firewalls, J. High Energy Phys. 06 (2013) 085.

[54] L. Susskind, Black hole complementarity and the HarlowHayden conjecture, arXiv:1301.4505.

[55] J. de Boer, S. F. Lokhande, E. Verlinde, R. van Breukelen, and K. Papadodimas, On the interior geometry of a typical black hole microstate, arXiv:1804.10580.

[56] P. Hayden and G. Penington, Learning the alpha-bits of black holes, arXiv:1807.06041.

[57] A. Almheiri, Holographic quantum error correction and the projected black hole interior, arXiv:1810.02055.

[58] D. N. Page, Is Black Hole Evaporation Predictable?, Phys. Rev. Lett. 44, 301 (1980).

[59] Y. Nomura, J. Varela, and S. J. Weinberg, Black holes, information, and Hilbert space for quantum gravity, Phys. Rev. D 87, 084050 (2013).

[60] S. Ghosh and S. Raju, Quantum information measures for restricted sets of observables, Phys. Rev. D 98, 046005 (2018).

[61] G. Dvali, Black holes and large N species solution to the hierarchy problem, Fortschr. Phys. 58, 528 (2010).

[62] J. B. Hartle and S. W. Hawking, Path integral derivation of black hole radiance, Phys. Rev. D 13, 2188 (1976).

[63] P. Hayden and J. Preskill, Black holes as mirrors: Quantum information in random subsystems, J. High Energy Phys. 09 (2007) 120.

[64] Y. Sekino and L. Susskind, Fast scramblers, J. High Energy Phys. 10 (2008) 065.

[65] J. M. Bardeen, Black Holes Do Evaporate Thermally, Phys. Rev. Lett. 46, 382 (1981).

[66] D. N. Page, Particle emission rates from a black hole: Massless particles from an uncharged, nonrotating hole, Phys. Rev. D 13, 198 (1976).
[67] W. Israel, Thermo field dynamics of black holes, Phys. Lett. 57A, 107 (1976).

[68] R. Bousso, Violations of the Equivalence Principle by a Nonlocally Reconstructed Vacuum at the Black Hole Horizon, Phys. Rev. Lett. 112, 041102 (2014).

[69] Y. Nomura, Physical theories, eternal inflation, and the quantum universe, J. High Energy Phys. 11 (2011) 063.

[70] J. Maldacena, D. Stanford, and Z. Yang, Diving into traversable wormholes, Fortschr. Phys. 65, 1700034 (2017).

[71] W. G. Unruh and R. M. Wald, What happens when an accelerating observer detects a Rindler particle, Phys. Rev. D 29, 1047 (1984).

[72] J. Maldacena, S. H. Shenker, and D. Stanford, A bound on chaos, J. High Energy Phys. 08 (2016) 106.

[73] H. Bondi, M. G. J. van der Burg, and A. W. K. Metzner, Gravitational waves in general relativity VII. Waves from axisymmetric isolated systems, Proc. R. Soc. A 269, 21 (1962).

[74] R. K. Sachs, Gravitational waves in general relativity VIII. Waves in asymptotically flat space-times, Proc. R. Soc. A 270, 103 (1962).

[75] M. Mirbabayi and M. Porrati, Dressed Hard States and Black Hole Soft Hair, Phys. Rev. Lett. 117, 211301 (2016).

[76] R. Bousso and M. Porrati, Soft hair as a soft wig, Classical Quantum Gravity 34, 204001 (2017).

[77] J. M. Bardeen, B. Carter, and S. W. Hawking, The four laws of black hole mechanics, Commun. Math. Phys. 31, 161 (1973).

[78] J. D. Bekenstein, Generalized second law of thermodynamics in black hole physics, Phys. Rev. D 9, 3292 (1974).

[79] Y. Nomura, P. Rath, and N. Salzetta, Spacetime from unentanglement, Phys. Rev. D 97, 106010 (2018). 